\title{
1 Biomaterials for 4D stem cell culture
}

2 Amber M. Hilderbrand ${ }^{1 *}$, Elisa M. Ovadia ${ }^{1 *}$, Matthew S. Rehmann ${ }^{1}$, Prathamesh M. Kharkar ${ }^{2}$,

3 Chen Guo ${ }^{1}$, April M. Kloxin ${ }^{1,2,+}$

$4 \quad *$ Both authors contributed equally to this work

$5 \quad{ }^{1}$ Department of Chemical and Biomolecular Engineering, University of Delaware, Newark, DE

6 19716, USA

7

$8{ }^{2}$ Department of Materials Science and Engineering, University of Delaware, Newark DE 19716, 9 USA

10

$11+$ Author for correspondence

12 Tel: $1302-831-3009$

13 Email: akloxin@udel.edu

14

15 Key words: hydrogels, stem cells, biomaterials, 3D cell culture, click chemistry

16

17 Submitting to Current Opinion in Solid State and Materials Science, 2015

18

19 Article Type: Review Article

20 Deadline: 14 August 2015

21 Format: 6-8 pages, $40-50$ references, $3-5$ figures 


\section{Abstract}

2 Stem cells reside in complex three-dimensional (3D) environments within the body that 3 change with time, promoting various cellular functions and processes such as migration and

4 differentiation. These complex changes in the surrounding environment dictate cell fate yet, until 5 recently, have been challenging to mimic within cell culture systems. Hydrogel-based

6 biomaterials are well suited to mimic aspects of these in vivo environments, owing to their high 7 water content, soft tissue-like elasticity, and often-tunable biochemical content. Further, 8 hydrogels can be engineered to achieve changes in matrix properties over time to better mimic 9 dynamic native microenvironments for probing and directing stem cell function and fate. This 10 review will focus on techniques to form hydrogel-based biomaterials and modify their properties 11 in time during cell culture using select addition reactions, cleavage reactions, or non-covalent 12 interactions. Recent applications of these techniques for the culture of stem cells in four 13 dimensions (i.e., in three dimensions with changes over time) also will be discussed for studying 14 essential stem cell processes. 


\section{Introduction}

Stem cells have two major defining characteristics: the ability 1) to self-renew, giving rise to multiple cells of the same type, and 2) to differentiate into tissue- or organ-specific cells upon receiving the proper cues [1]. These fundamental and essential attributes give stem cells the potential to regenerate or heal tissues throughout the body. Understanding and controlling the underlying cues that direct stem cell renewal and differentiation is key to unlocking this potential. Insight into these processes can be gained from examination of native stem cell environments and applied to the design of synthetic dynamic microenvironments for controlled expansion and culture of stem cells.

In the body, stem cells reside in specific microenvironments composed of extracellular matrix (ECM), cells resident or recruited to the tissue, and cell-secreted factors, such as growth factors and cytokines [2]. These microenvironments provide dynamic extracellular biophysical and biochemical cues that influence stem cell processes, including proliferation, migration, and differentiation (Figure 1A). For example, three-dimensional (3D) stem cell niches have a range of mechanical properties, including 'soft' neural tissues containing neural progenitor cells (E 0.1-1 kPa), bone marrow containing mesenchymal and hematopoietic stem cells $(\mathrm{E} \sim 0.5 \mathrm{kPa})$, and more elastic muscle tissue containing satellite cells $(\mathrm{E} \sim 10 \mathrm{kPa})$ [3]. Large ECM proteins, such as collagens, fibronectin, and laminins, and glycosaminoglycans, such as hyaluronic acid (HA), heparan sulfate, and chondroitin sulfate, provide structural and biochemical content comprising the base matrix in which stem cells reside [4]. Stem cells bind to these ECM proteins through receptors, especially integrins that regulate processes such as cell adhesion and signal transduction. Additional biological cues often sequestered by the ECM, such as growth factors, cytokines, and morphogens, also support cellular processes such as proliferation and 
1 differentiation within the stem cell niche [5]. For example, differentiation of stem cells into

2 dopaminergic neurons, the cell type most affected by Parkinson's disease, is caused by gradients

3 of WNT and sonic-hedgehog (SHH) morphogens during development [6].

$4 \quad$ Hydrogels, crosslinked hydrophilic polymer networks, provide a platform for mimicking

5 key in vivo characteristics of the microenvironments surrounding stem cells. Used as matrices for

6 3D cell culture, hydrogels enable decoupling of the complex milieu of cues found within the

7 native ECM for simplified, yet physiologically-relevant, cell culture studies that mimic important

8 aspects of the native environment while facilitating hypothesis testing. Natural hydrogels are

9 typically composed of naturally derived biological components, such as type I collagen,

10 hyaluronic acid, or basement membrane extract (BME), and capture some of the supramolecular

11 structures and biological content of the native ECM. However, control of matrix elasticity or

12 'stiffness' and biological cues can be restricted within strictly natural hydrogels, owing to their

13 inherent protein structure and chemistry and batch-to-batch variation, which may limit their use

14 for hypothesis testing [7]. To address this, synthetic and natural polymers have been modified

15 with reactive functional groups to form synthetic and hybrid hydrogels that provide more user

16 control and a wider range of properties, creating synthetic ECMs for the controlled, 3D culture of 17 cells over time.

18 Hydrogel-based synthetic ECMs have been designed to provide user control of gel 19 formation, degradation, or other features such as addition of biochemical moieties (e.g., peptide 20 tethers and crosslinks). Initially, hydrolytic degradation was incorporated within such materials

21 to impart temporal changes in properties based on monomer design, allowing preprogrammed

22 decreases in matrix crosslink density or removal of biochemical moieties by cleavage of 23 crosslinks or pendant groups, respectively. For example, Burdick and coworkers functionalized 
1 hyaluronic acid (HA) with methacrylate groups and varied the number of ester bonds between

2 the HA and the methacrylate group to control the rate of degradation and thereby the crosslink

3 density of the resulting hybrid hydrogels over time for the culture of mesenchymal stem cells

4 (MSCs). Increasing the number of ester repeat units increases the probability and rate of

5 hydrogel degradation [8]. To impart responsive cell-driven control of matrix properties over

6 time, a suite of materials that are enzymatically degraded have been developed. Incorporation of

7 enzymatically degradable crosslinkers or tethers (e.g., matrix metalloproteinase (MMP)

8 cleavable peptides) allows remodeling of synthetic ECMs by enzymes secreted by stem cells at

9 specific times during their life cycle toward capturing the in vivo process of tissue remodeling.

10 Bian and coworkers incorporated MMP degradable peptides into hydrogels formed with

11 methacrylated HA to allow cell-mediated degradation, supporting cell culture for more than 14

12 days. Within these gels the human mesenchymal stem cells (hMSCs) showed enhanced

13 chondrogenesis and suppressed hypertrophy [9].

14 Over the past few years, synthetic matrices have been expanded to incorporate multiple

15 forms and levels of hydrogel property control (e.g., combinations of cleavage and addition

16 reactions for temporal modulation of synthetic ECMs) toward more accurately mimicking in vivo

17 stem cell microenvironments. Broadly, 4D biomaterials, or 3D matrices whose properties change

18 over time, enable the study of dynamic stem cell-microenvironment interactions relevant to

19 healthy and diseased tissues in vitro (Figure 1B) [4,10]. This review will focus on how

20 hydrogel-based biomaterials have been designed to recapture aspects of dynamic, 3D in vivo

21 microenvironments to study important functions and fates of MSCs, embryonic stem cells

22 (ESCs), induced pluripotent stem cells (iPSCs), and progenitor cells (i.e., cells that differentiate

23 into a specified cell lineage). The following sections will summarize general design 
1 considerations for 4D material properties depending on the target application (Section 2.1),

2 varieties of 'click' chemistries to form or modify these materials during cell culture (Section

3 2.2), mechanisms of degradation and non-covalent interactions and how they have been used to

4 achieve a more accurate mimic of cell microenvironments over time (Section $2.3-2.4$ ), and 5 applications of these 4D biomaterials for the culture of stem cells in changing 6 microenvironments (Section 3).

\section{2. Chemistries to form and modify hydrogels}

8 This section will overview properties to consider when designing materials for stem cell

9 culture along with hydrogel formation techniques to create materials that mimic key aspects of 10 native microenvironments in vitro. Reactions and mechanisms for modifying these materials and

11 their selection for achieving desired material properties also will be discussed. These include 12 click chemistry reactions and non-covalent chemistries to sequentially form and modify 13 hydrogels and relevant methods to degrade hydrogels in a controlled manner in the presence of 14 cells (Figure 2).

\section{$15 \quad 2.1$ General design considerations}

16 A variety of parameters must be considered when designing materials for stem cell

17 culture, including cytocompatibility, transport properties, and ways to quantify cell response to

18 material changes. First and foremost, the base polymers and chemistries must maintain and

19 support cell viability during hydrogel fabrication, modification, and culture. The mechanism for

20 forming materials in the presence of cells for 3D culture should not result in significant cell

21 death, DNA damage, or expression of cell stress markers (e.g., p53). With this in mind, the

22 chemistries discussed below (sections 2.2 and 2.3) all have been executed in the presence of

23 cells. Further, the resulting material must have an appropriate pore size (roughly $>5 \mathrm{~nm}$ ) to 
1 allow for nutrient, waste, and soluble factor (e.g., growth factors) diffusion within the matrix, as

2 well as potential diffusion of biochemical reagents (e.g., antibodies or peptides) introduced for

3 imaging or modification reactions [11,12]. To quantify cell responses to changes in matrix

4 properties, materials must allow for various measurements and assays of biological functions.

5 For cell imaging, a material that is optically clear or does not interfere with light transmission is

6 beneficial. For techniques like flow cytometry or qRT-PCR to look at receptor or gene

7 expression, methods for degradation of the hydrogel under mild conditions (section 2.3) that

8 maintain surface receptors and retention of cell phenotype may be required or useful to recover

9 the cells for analysis.

$10 \quad 2.2$ Formation and addition reactions

11 Click reactions are an attractive set of chemistries to form materials for cell culture

12 owing to their versatility, often fast reaction kinetics, and their ability to proceed in mild

13 conditions (e.g., room temperature aqueous). In addition, several bioorthogonal click reactions

14 have been established that can be used during cell culture without interference with natural

15 cellular processes. Owing to these beneficial properties, a variety of click reactions have been

16 used to form and add elements to materials in the presence of stem cells for 4D culture (Figure $17 \quad 2 A)$.

18 2.2.1 Azide-alkyne reactions

19 Strain promoted azide-alkyne cycloaddition (SPAAC) is a widely used bioorthogonal

20 reaction that proceeds at reasonable rates in aqueous solutions without a catalyst to form a

21 triazole linkage [13]. Traditional copper-catalyzed azide-alkyne cycloaddition has proven useful

22 for the fabrication of materials outside of a biological context [14]; however, the Copper (I)

23 catalyst is cytotoxic, and consequently SPAAC has been investigated for the formation of 
1 biomaterials in the presence of cells [15]. For example, Becker and coworkers showed $>95 \%$

2 viability of human mesenchymal stem cells 24 hours after encapsulation in hydrogels formed

3 with 4-dibenzocyclooctynol-functionalized, 4-arm poly(ethylene glycol) (PEG) crosslinked with

4 a 3-arm glycerol exytholate triazide [16]. This class of reactions typically has been used to form

5 hydrogels while other types of addition or cleavage reactions are used for modification, owing to

6 the orthogonality of the SPAAC reaction with respect to these other reactive functionalities.

\section{$7 \quad$ 2.2.2 Thiol-ene and Thiol-yne reactions}

Thiol-ene reactions occur between a thiol and an alkene group such as a norbornene,

9 acrylate, or vinyl sulfone [17]. They have been reacted by radically-initiated polymerizations or

10 Michael-type nucleophilic additions depending on the functional group (e.g., norbornenes or

11 vinyl sulfones, respectively) and reaction conditions (e.g., photoinitiation or basic $\mathrm{pH} / \mathrm{catalyst}$ for

12 acrylates, respectively). Free radical initiation results in a thiyl radical that adds to the

13 unsaturated 'ene', forming a thioether bond and regenerating the radical for further reaction [17].

14 In Michael-type addition of thiols to alkenes, a nucleophile adds to an electron deficient vinyl

15 group generating a base, which deprotects and generates a thiolate anion initiating the addition

16 cycle [14]. For example, Burdick and coworkers encapsulated human mesenchymal stem cells

17 with good viability (88\%) in HA hydrogels using norbornene-functionalized HA and a dithiol

18 crosslinker. A second thiol-ene reaction of excess norbornene groups with either additional

19 dithiol crosslinker, or thiol functionalized dyes was used to modify the network, increasing the

20 crosslink density at specific positions within the hydrogel or adding pendant groups using 21 photopatterning [18].

22 Thiols also react with alkyne groups by a similar radically-mediated mechanism. In

23 forming a polymer network, a 2:1 ratio of thiol to alkyne leads to the sequential propagation of a 
1 thiyl radical with an alkyne or with a vinyl functional group that was generated by the former

2 reaction [19]. Since two thiols can be reacted with one alkyne, the thiol-yne reaction provides a

3 way to increase network connectivity relative to thiol-ene systems without changing the number

4 of functional groups per monomer. However, one drawback is the differential reactivity of

5 alkyne functional groups, where some do not allow a second thiol addition [19]. Dove and

6 coworkers used simultaneous application of nucleophilic thiol-yne reaction and inverse electron-

7 demand Diels-Alder additions to create an interpenetrating network with robust mechanical

8 properties (withstood compressive stresses of 4-15 MPa) that maintained cell viability (48 hours

9 after encapsulation) [20]. They also employed thiol-ene addition and tetrazine ligation reactions

10 to pattern pendant groups into the matrix over time (a fluorescent dye with a terminal thiol group

11 [BODIPY-SH] and a tetrazine-functionalized biotin), while maintaining excellent hMSC

12 viability ( $>99 \%$ after patterning). Broadly, thiol-ene and thiol-yne reactions offer

13 cytocompatible methods to both form and modify hydrogels in situ with a diverse suite of

14 functional group choices in 'enes', 'ynes', and thiols, allowing users to easily tailor material

15 properties.

\section{$16 \quad 2.2 .3$ Diels-Alder cycloaddition}

17 Diels-Alder reactions are widely used for hydrogel formation by reacting conjugated

18 dienes with substituted alkenes. In general, these reactions proceed under mild conditions

19 without the need for an initiator and have been used to form hydrogels for cell encapsulation

20 [21]. Note, the kinetics of gelation can be slow relative to other types of gelation mechanisms,

21 such as photoinitiation, taking place on the order of hours depending on the functional groups

22 selected. Chen and coworkers recently formed hydrogels using hyaluronic acid modified with

23 furan and poly(ethylene glycol) functionalized with maleimide groups leaving an available 
1 double bond for subsequent reaction after gel formation [22]. Hydrogel moduli between $\sim 4-75$

$2 \mathrm{kPa}$ were achieved, comparable to the elasticity of various soft tissues including brain, fat, and

3 muscle [22]. A subsequent thiol-ene reaction with a FITC-labeled RGDSC peptide was used to

4 pattern the hydrogel, and no significant differences in mechanical properties were observed

5 before and after patterning, providing independent control of biophysical and biochemical cues.

\section{$6 \quad$ 2.2.4 Oxime ligation}

7 Reactions between aminooxy functional groups and aldehydes or ketones produce imine

8 bonds. These reactions occur readily in aqueous solutions, and side reactions are minimal [23]. A

9 catalyst is not always required but may be used to increase reaction rate. For example, Becker

10 and coworkers showed that, by using an aniline catalyst and controlling $\mathrm{pH}$, gelation time was

11 reduced (hours to seconds) and storage modulus increased ( $0.3 \pm 0.1 \mathrm{kPa}$ to $4.7 \pm 0.3 \mathrm{kPa})$ for

12 hydrogels formed by reaction of aldehyde-functionalized PEG with a 4-arm aminooxy

13 crosslinker [24]. Further, the authors used a combination of oxime, azide-alkene, and

14 photoinitiated thiol-ene click reactions to create patterned hydrogels, mimicking changes in

15 biochemical content within the niche surrounding cells.

\section{$16 \quad 2.3$ Cleavage reactions}

17 Cleavage reactions generally present an attractive strategy to change biomaterial

18 properties in a controlled manner as a function of time, including mechanical (i.e., decrease in

19 crosslinking density) and biochemical properties (i.e., incorporation or release of peptides or

20 cytokines) to capture aspects of dynamic cell microenvironments (Figure 2B). This section will

21 focus on key examples that utilize cleavage reactions in designing dynamic 4D hydrogels for

22 stem cell culture. For a comprehensive review of degradable chemistries, readers are referred to a

23 review by Kharkar and coworkers [25]. 


\subsubsection{Ester hydrolysis}

Traditionally, ester hydrolysis has been used for predetermined, continuous changes in biomaterial properties. Ester linkages cleave in aqueous conditions to form a carboxylic acid and an alcohol. The rate of cleavage ranges from a few days to a few years depending on the number of ester linkages, the functional groups surrounding the ester linkages, the network hydrophobicity, and the surrounding microenvironment $\mathrm{pH}[25,26]$. In $4 \mathrm{D}$ hydrogels that incorporate ester linkages, the rate of cleavage or network degradation is usually preprogrammed based on the number and type of ester bonds incorporated during monomer and hydrogel formation [8,27]. For example, Lin and coworkers reported the use of PEG-based synthetic hydrogels containing different amounts of hydrolytically-degradable thiol-ether-ester linkages for 4D hMSC culture and differentiation [27]. Hydrogels were formed using visible lightinitiated mixed mode polymerization of acrylates and thiols in the presence of the photoinitiator Eosin Y. The ester linkage underwent hydrolysis with different pseudo-first-order rate constants ( $\mathrm{k} \sim 0.02$ to $\sim 0.17 \mathrm{day}^{-1}$ ) depending on the number of degradable crosslinks (controlled wt\% of thiol crosslinker concentration). When encapsulated within fast-degrading hydrogels $(\mathrm{k}=0.17$ day $^{-1}$ ), hMSCs showed a higher degree of osteogenic differentiation compared to slow-degrading hydrogels $\left(\mathrm{k}=0.03 \mathrm{day}^{-1}\right)$. Degradation allowed for increased cell spreading and deposition of cell-secreted proteins, promoting osteogenic differentiation.

\subsubsection{Enzymatic cleavage reactions}

Hydrogels can undergo enzymatic hydrolysis when the material is constructed using a naturally-derived polysaccharide (i.e., hyaluronic acid, collagen) or enzyme-sensitive peptide linkage (i.e., MMP-cleavable linkages) [28,29]. The concentrations of enzymes that selectively degrade the hydrogel backbone dictate the rate and extent of cleavage. Enzyme concentrations, 
1 and consequently gel degradation, have been controlled by exogenous addition or secretion by

2 cells within these MMP-degradable hydrogels. In particular, enzymatically degradable hydrogels

3 have been used to introduce changes within synthetic matrices to study stem cell response in

4 dynamic microenvironments [9,30,31]. For example, Burdick and coworkers reported the use of

5 hyaluronic acid based hydrogels that undergo degradation by enzymatic hydrolysis in addition to

6 ester hydrolysis for the culture of encapsulated hMSCs. Changes in the matrix structure and

7 modulus, due to cell-driven degradation of the network, resulted in upregulation of type II

8 collagen and aggrecan, indicating chondrogenesis. This work demonstrated the importance of 4D

9 biomaterials for lineage-specific differentiation, particularly for cartilage regeneration

10 applications [30].

\section{2.3.3 Light-mediated cleavage reactions}

The use of photolabile functional groups that cleave under cytocompatible doses of light,

14 such as o-nitrobenzyl ethers and coumarins, allows user-directed control over biomaterial

15 properties at times and positions of interest [32-34]. The $o$-nitrobenzyl ether group undergoes

16 irreversible rearrangement and cleavage upon irradiation (long-wavelength UV, visible, or two

17 photon light), yielding a ketone and carboxylic acid. Hydrogels formed using these photolabile

18 groups have been used to investigate stem cell response to dynamic material changes, as well as

19 to control release and retention of different biological cargoes (e.g., growth factors or stem cells

20 for therapeutic applications) [35-37]. For example, in parallel complementary studies, Anseth

21 and coworkers and Kasko and coworkers encapsulated human mesenchymal stem cells within

22 synthetic 4D hydrogels containing photodegradable $o$-nitrobenzyl ether group variants [36,38].

23 By cleavage with specific wavelengths of light, the encapsulated cells were spatiotemporally

24 released from hydrogels using photoerosion while maintaining cell viability (>95\%). 
Coumarin linkages also undergo degradation with cytocompatible light doses to yield alcohol

2 and carboxylic acid functional groups, with the rate of cleavage varying based on aromatic ring

3 substituents. Polymeric hydrogels containing coumarin groups have potential for use in 4D

4 culture [34,39]. For example, Almutairi and coworkers reported the synthesis of ornithine-based

5 bromohydroxycoumarin crosslinker to form polyacrylamide hydrogels [40]. Controlled release

6 of encapsulated stem cells was triggered by long wavelength UV light $\left(10 \mathrm{~mW} / \mathrm{cm}^{2}\right.$ at $365 \mathrm{~nm}$

7 for 15 minutes) demonstrating its utility for design of a 4D hydrogel matrix and for cell delivery.

8 Light also has been used to control the release of calcium or calcium chelators in alginate

9 hydrogels for temporally modulating hydrogel crosslink density and modulus [41]. Calcium (or a

10 calcium chelator) and gold nanorods were encapsulated in thermal responsive 1,2-dipalmitoyl-

11 sn-glycero-3-phosphocholine liposomes with a gel-to-fluid transition temperature of $41^{\circ} \mathrm{C}$. When

12 irradiated with near-infrared light, the gold nanorods locally heated the surrounding fluid due to

13 surface plasmon resonance, disrupting the liposomes. This light-mediated release of calcium

14 crosslinker or calcium chelator resulted in stiffening ( $\sim 90 \mathrm{~Pa}$ to $\sim 1200 \mathrm{~Pa}$ ) or softening ( 900 Pa

15 to $\sim 400 \mathrm{~Pa}$ ) of the hydrogels, respectively. Such an approach can be translated to cell-laden

16 hydrogels to study changes in stem cell function and fate in response to dynamic changes in 17 matrix density.

18 While only single modes of degradation were discussed here (section 2.3), combinations

19 of two or more cleavage reactions have been utilized to study stem cell behavior and fate as

20 discussed later (section 4) [27,30,42]. Additionally, other cleavage or reversible reactions of note

21 yet absent here, including retro Diels-Alder reactions [43], retro Michael-type reactions [44-46],

22 azobenzene photoisomerization [47], and disulfide cleavage [42], are promising but less explored

23 to date in the design of 4D biomaterials for stem cell research. 
Unlike several of the aforementioned covalent gelation mechanisms, non-covalent

3 interaction-induced gelation typically occurs by self-assembly without a need for initiator or

4 catalyst. The lack of external chemical components makes these mechanisms often

5 cytocompatibile and well-suited for use in the presence of cells, provided the interaction strength

6 is sufficient. Commonly for 3D culture, peptides and proteins have been designed to assemble

7 through non-covalent interactions, including hydrogen bonding, aromatic stacking, ionic, and

8 hydrophobic interactions [7,48-55], to form hydrogels upon mixing (Figure 2C). In this section,

9 we will give an overview of hydrogels formed upon mixing of engineered peptides or proteins,

10 including those formed with two components or by applying a shear force, and other polymeric

11 hybrid hydrogels for controlled stem cell culture applications.

\section{2.4.1 Peptide hydrogels}

13 Peptides have been designed with various strong non-covalent interactions to promote

14 assembly and hydrogel formation under physiological conditions. For example, Kokkoli and

15 coworkers investigated a fibronectin-mimetic peptide amphiphile, $\mathrm{C}_{16^{-}}$

16 GGGSSSPHSRN(SG) $)_{5}$ RGDSP (PR_g), which assembles in water to form nanofibrous

17 hydrogels. On the PR_g peptide sequence, RGD provides the primary cell binding motif with the

18 PHSRN sequence included as the synergy site to impart specificity and affinity for the integrin

$19 \alpha 5 \beta 1$. PR_g peptide amphiphiles were diluted with another peptide, $\mathrm{C}_{16}$-GGGSSSESE (E $\mathrm{E}_{2}$ ), to

20 screen charges and enable faster gelation. PR_g/E2 gels supported 3D culture and proliferation

21 of fibroblasts (NIH3T3/GFP) over five days [53]. In seminal work, Stupp and coworkers

22 designed another peptide amphiphile (PA) that contained a 16-carbon chain, 4 alanine residues, 3

23 glycine residues, and the sequence IKVAV, which is found in laminin and known to promote 
1 and direct neurite outgrowth. Self-assembly was induced by electrostatic repulsions between the

2 alanines and glycines, hydrogen bond formation, and the unfavorable contact between

3 hydrophobic segments and water. Murine neural progenitor cells (NPCs) were encapsulated in

4 these IKVAV-PA gels, and cell differentiation and migration were observed [54].

$5 \quad$ Using a similar amphiphilic building block concept, Ulijn and coworkers designed

6 fluorenylmethoxycarbonyl (Fmoc) dipeptides that formed hydrogels by hydrogen bonding and

7 aromatic stacking. After studying a library of seven Fmoc-dipeptides, the Fmoc-phenylalanine

8 dipeptide, as well as mixtures of this sequence with Fmoc-glycine-glycine or Fmoc-lysine,

9 formed hydrogels at physiological $\mathrm{pH}$. These three gels supported chondrocyte proliferation in

$103 \mathrm{D}$ culture [55]. More broadly, these examples provide design principles for the creation of

11 dynamic, physical hydrogels that enable 3D cell culture.

12 Approaches to regulate peptide assembly provide handles for controlling hydrogel

13 formation or modulating properties. For example, Pochan, Schneider, and coworkers developed

14 MAX8 hydrogels using a 20 amino acid peptide that self-assembles through electrostatic

15 repulsions, hydrophobic contacts, hydrophobic collapse, and hydrogen bonding [48]. This

16 hydrogel shear thins when a force is applied, disrupting the interactions and allowing injection or

17 manipulation of the now-liquid solution; however, once the force is released, the interactions are

18 reestablished, and the hydrogel regains its structural integrity. With this approach, a

19 mesenchymal stem cell line (C3H10t1/2) was encapsulated within these hydrogels, indicating

20 their cytocompatibility. Shear-thinning systems such as this also are useful for delivery of cells

21 to target biological sites.

22 Utilizing two sequences for assembly is another strategy for controlling hydrogel

23 formation. For example, Butler and coworkers developed hydrogelating self-assembling fibers 
1 (hSAFs) composed of two 28-residue peptides of the coiled-coil heptad sequence repeat

2 (sequence I: K IAALKAK IAALKAE IAALEWE NAALEA and sequence II: K IAALKAK

3 NAALKAE IAALEWE IAALEA) that form an $\alpha$-helical dimer. The gelation mechanism of

4 these sequences for hydrogel formation was manipulated by carefully choosing amino acids for

5 each position in the heptad repeat, which dictated whether hydrogen bonding or hydrophobic

6 interactions would occur. In particular, a two-component peptide solution was established for the

7 formation of a self-supporting hydrogel through hydrophobic interactions that supported the

8 growth and differentiation of a model neuronal-like cell line (rat adrenal pheochromocytoma

9 [PC12] cells). As a dual-component system, these hSAFs provide increased control of gelation

10 time through mixing [49].

\section{$11 \quad 2.4 .2$ Protein and 'hybrid' hydrogels}

12 Proteins also have been designed for physical interactions and cell-binding to enable 3D

13 cell culture. For example, Heilshorn and coworkers created a mixing-induced two-component

14 hydrogel (MITCH) where the two components form a network through hydrogen bonding of

15 complementary peptide domains. The two recombinant protein components, C7 and P9,

16 contained 7 and 9 repeats of complementary binding domains, which physically crosslinked to

17 form hydrogels within seconds. Human adipose derived stromal cells (hADSCs) were

18 encapsulated in these hydrogels, along with FGF-1 and BMP-4, to demonstrate the potential use

19 as in vitro models of disease progression, drug delivery, and in vivo tissue engineering [50].

20 More details on the stem cell studies using this MITCH system are discussed in section 4.

21 Block copolymer hydrogels also have shown promise for stem cell culture applications.

22 Ding and coworkers demonstrated a facile poly( $\varepsilon$-caprolactone-co-lactide)-poly(ethylene glycol)-

23 poly(E-caprolactone-co-lactide) (PCLA-PEG-PCLA) ABA triblock copolymer hydrogel 
1 formation, exhibiting a sol-gel transition between room $\left(25^{\circ} \mathrm{C}\right)$ and body temperature $\left(37^{\circ} \mathrm{C}\right)$.

2 Physical gelation occurred due to the formation of a percolated micelle network. RGD was 3 covalently linked to the hydrophobic PCLA or hydrophilic PEG block to promote cell adhesion,

4 specifically for studying chondrocyte function. Peptide incorporation facilitated ligand-integrin

5 interactions and improved cell viability [56]. Additionally, Schaffer and coworkers demonstrated

6 the use of poly(N-isopropylacrylamide)-co-poly(ethylene glycol) (PNIPAAm-PEG) for 3D cell

7 culture of hPSCs utilizing the inverse solubility of the PNIPAAM block upon heating to form

8 and subsequently dissolve hydrogels during culture and expansion of hPSCs [57]. This example

9 will be discussed further in section 3.2.1. Combining polymeric and assembling peptide systems,

10 Chmielewski and coworkers developed an 8-arm 'block copolymer' where each arm contains

11 one block of PEG and one block of collagen mimetic peptide (CMP) with 8 repeats of the 12 proline-hydroxyproline-glycine $\left[(\mathrm{POG})_{8}\right]$. The $\mathrm{CMP}$ block formed a triple helix through

13 hydrogen bonding, which served as physical crosslinks and enabled hydrogel formation. hMSCs

14 were encapsulated in these hydrogels and remained viable for more than 5 days [52].

15 The noncovalent interactions presented here to date mainly have been utilized for the

16 initial formation of hydrogels. These chemistries lead to physical crosslinks that are inherently

17 dynamic and, in principle, allow in situ rearrangement of linkages based on microenvironment

18 conditions (e.g., salt concentration, applied force, or other relevant stimuli). Notably, these

19 reactions generally are orthogonal to many covalent addition and cleavage reactions and have the

20 potential to be combined with these two groups of reactions to better mimic temporal changes in

21 the ECM for stem cell culture applications.

\section{3. 4D biomaterials for stem cell cultures}


Biomaterials that enable 3D cell culture and changes in their biophysical or biochemical

2 properties over time (i.e., in the fourth dimension) provide tunable microenvironments for stem

3 cell culture and control of cues for modulating stem cell behavior. These materials mimic aspects

4 of conditions encountered in vivo for probing stem cell differentiation while supporting cell

5 viability, proliferation, and spreading, as well as enabling the delivery of cells and growth factors

6 for translating these findings to therapeutic treatments. This section will discuss the utilization of

7 4D biomaterials for the culture of MSCs, ESCs, iPSCs, and progenitor cells and stem-cell

8 derived cells.

\section{$9 \quad 3.1$ Mesenchymal Stem Cells}

10 Mesenchymal stem cells are plastic-adherent cells from the bone marrow and other

11 tissues that are capable of differentiating into osteoblasts, adipocytes, and chondrocytes, amongst

12 other lineages [58]. MSCs offer tremendous therapeutic potential owing to their multilineage

13 differentiation ability, secretion of large amounts of growth factors and other proteins, homing to

14 injured tissues, and suppression of immune responses from surrounding cells [59]. As a result,

15 they have been the focus of many recent studies involving 4D hydrogel-based biomaterials,

16 which are used to modulate MSC adhesion, viability, migration, spreading, and differentiation.

\section{$17 \quad$ 3.1.1 MSC viability and spreading}

18 One of the major ways MSCs interact with their ECM (and biomaterials that mimic the

19 ECM) is through adhesive ligands present in ECM proteins. These ligands promote MSC

20 survival by allowing adhesion to the matrix and can promote cell signaling to direct cell function,

21 especially through integrins [60]. For 4D biomaterial studies, MSC adhesion can be promoted

22 either by incorporation of ECM proteins or synthetic peptide mimics into the biomaterial

23 network (or, in the case of natural materials, already present in the network). For example, 
1 Anseth and coworkers, amongst many others, have shown that MSCs adhere, survive, and spread

2 when cultured in three-dimensions in enzymatically-degradable PEG networks functionalized

3 with the adhesive ligand RGDS, a common motif found in many proteins that binds to various

4 integrins including $\alpha_{v} \beta_{3}$ and $\alpha_{5} \beta_{1}$, which are strongly associated with vitronectin and fibronectin,

5 respectively [61]. These networks were formed by a photoinitiated thiol-ene reaction of a multi-

6 arm PEG-norbornene monomer and an enzymatically-degradable peptide (GPQG $\downarrow$ IWGQ; arrow

7 denotes cleavage site) that cells cleave through MMP secretion, mimicking in vivo remodeling of

8 the ECM. DeForest and Tirrell employed a photoreversible protein patterning approach to

9 control MSC spreading in specific locations during cell culture in response to the protein

10 vitronectin [62]. The authors formed PEG-based networks by SPAAC (Figure 3A) and

11 incorporated a photocaged alkoxyamines within the network. After network formation and 12 subsequent irradiation with a cytocompatible dose of long wavelength UV light, the

13 alkoxyamines were uncaged for reaction with aldehyde-functionalized proteins by oxime

14 ligation, permitting spatiotemporal control of protein presentation within the network. Further,

15 the aldehyde moiety was functionalized with a photodegradable $o$-nitrobenzyl ether group,

16 allowing removal of the tethered protein-based cue from the network. MSCs were only able to

17 adhere and spread in the network in the presence of vitronectin, and this interaction promoted

18 MSC osteogenic differentiation only in regions of the gel where vitronectin was present (Figure 19 3B).

\section{3.1.2 MSC differentiation}

21 The regulation and study of MSC differentiation is coupled with the study of adhesion,

22 since many cues that regulate adhesion and spreading also impact MSC fate, and a variety of 23 dynamic materials have been developed to study and direct MSC differentiation. For example, 
1 Johnstone and coworkers developed an MMP-7-responsive PEG-based hydrogel that enhanced

2 chondrogenesis of MSCs [63]. MMP-7-sensitive peptide substrates (PLE $\downarrow$ LRA and

3 VPLS $\downarrow$ LTMG) were built with GGK at the C-terminus, so that both the $\mathrm{N}$-terminus and C-

4 terminus had accessible amines for modification. The pendant amines of these peptides were

5 conjugated with acrylate-PEG-succinimidyl carboxymethyl to form the macromer acrylate-PEG-

6 peptide-PEG-acrylate, which was polymerized by free radical chain polymerization

7 photoinitiated with Eosin Y to form an MMP-7-sensitive PEG hydrogels. MSCs encapsulated

8 within these materials produced a more extensive collagen matrix and formed stronger

9 neocartilage constructs as compared to a non-degradable control. Matrix degradation is not only

10 critical for protein production but also cell spreading and traction. Burdick and coworkers

11 explored the effect of degradation-mediated cellular traction on MSC differentiation by using a

12 4D hydrogel system [31]. Methacrylated HA was reacted with a difunctional MMP-crosslinking

13 peptides to form a HA-based matrix that cells could remodel, permitting MMP-mediated

14 degradation, MSC spreading, and osteogenic differentiation. To probe the role of matrix

15 degradability in differentiation, a secondary photopolymerization was used to chain-polymerize

16 free methacrylates on HA; this secondary polymerization limited the ability of MSCs to degrade

17 the HA hydrogel, preventing cell spreading and promoting adipogenic differentiation (Figure

18 3C). Notably, secondary crosslinking also was applied temporally after the MSCs had been

19 cultured for 7 days and already spread; the spread MSCs now entrapped within a non-degradable

20 matrix differentiated primarily down the adipogenic lineage. Taken together, these results

21 indicate that MSC fate within covalently crosslinked materials is dictated by the ability of MSCs

22 to degrade the matrix and generate traction forces, rather than by cell spreading (Figure 3D). 
Dynamic hydrogels also have been used to release growth factors to mediate MSC

2 differentiation. For example, Alsberg and coworkers formed cell-degradable gelatin

3 microspheres loaded with TGF- $\beta 1$ [64]. When the microspheres were cultured with high density

4 MSC aggregates, the MSCs secreted enzymes that degraded the gelatin, triggering release of the

5 growth factor. Localized growth factor release enhanced the chondrogenesis of MSCs within the

6 aggregates. Heilshorn and coworkers used a peptide-based MITCH system containing alginate

7 microgels to promote adipogenic differentiation of adipose-derived stem cells, which meet many

8 of the criteria for MSCs [50,65]. The authors encapsulated adipose-derived stem cells, the

9 growth factor FGF-1, and microspheres containing the growth factor BMP-4 within their

10 hydrogels. This strategy resulted in immediate exposure of the adipose-derived stem cells to

11 FGF-1 and delayed exposure to BMP-4 as it was released by hindered diffusion from the alginate

12 microspheres; this sequential growth factor release enhanced adipogenesis compared to

13 simultaneous presentation of either or both growth factors.

\section{1 .3 MSC migration and delivery for therapeutic applications}

15 Another major opportunity for therapeutic use of MSCs comes from their ability to

16 migrate to injured tissues, including the brain, heart, and lung, after injection or injury [66].

17 Hydrogel-based ECM mimics facilitate studying this process in vitro toward a greater

18 fundamental understanding of the mechanism and regulators of MSC migration. For example,

19 Lutolf and coworkers formed hydrogels from multi-arm PEG vinyl-sulfone, the MMP-

20 degradable peptide GCRDGPQG $\downarrow$ IWGQDRCG, and a substrate for the enzyme factor XIIIa that

21 was protected by a light-sensitive cage (FK(Nvoc)GGERCG) [67]. By irradiating the hydrogel

22 with a $30 \mathrm{~mW} 405 \mathrm{~nm}$ diode laser, the authors were able to site- and time-specifically cleave the

23 Nvoc protecting group, making the lysine accessible for conjugation to recombinantly-expressed 
1 proteins containing the other factor XIIIa substrate (NQEQVSPL). The authors used this

2 photouncaging approach to add RGD, a recombinant fibronectin fragment, and platelet derived

3 growth factor B, each engineered to contain the NQEQVSPL substrate, to specific regions of the

4 hydrogel. In each case, significantly greater MSC migration was observed in the patterned

5 regions than the unpatterned regions. Anseth and coworkers characterized human MSC

6 migration through hydrogels formed with multi-arm PEG monomers and MMP-degradable

7 crosslinking peptides (KCGPQG $\downarrow$ IWGQCK) [68]. Microrheology was used to quantify cell-

8 matrix interactions during cell remodeling. The authors showed that the hydrogel was degraded

9 in areas relatively far from the cell, due to the fact that the diffusion of cell-secreted MMPs is

10 faster than MMP cleavage of the substrate. More broadly, this study provides insight into the

11 mechanism of MSC migration within covalently crosslinked, cell-degradable hydrogels: MSCs

12 actively degrade the region surrounding the cell at short timescales (tens of minutes after

13 encapsulation), whereas, at longer timescales, the region surrounding the cell is degraded,

14 leading to an increased MSC migration rate.

15 Rather than having MSCs migrate to injured tissues, 4D biomaterials also have been

16 designed to locally release MSCs. Kasko and coworkers co-polymerized PEG-based hydrogels

17 with monomers containing different $o$-nitrobenzyl ether groups that exhibit different rates of

18 photocleavage and, consequently, hydrogel degradation [36]. By taking advantage of differences

19 in the rates of degradation, the authors temporally controlled the release of two distinct

20 populations of viable MSCs (expressing different fluorescent proteins). In another approach to

21 MSC delivery, Mooney and coworkers encapsulated rapidly degrading, hydrolytically-sensitive

22 alginate porogens and MSCs within slowly degrading, high molecular weight alginate hydrogels

23 [69]. During culture, the porogens degraded, creating interconnected pores within the high 
1 molecular weight alginate hydrogels that enabled cell migration and release. Complete MSC

2 release could be tuned from $\sim 5$ to 60 days based on porogen design parameters. This hydrogel

3 was applied in a rat model for the delivery of MSCs to cranial defect sites; hydrogels with an

4 elastic modulus (E) of $\sim 60 \mathrm{kPa}$ led to the most effective bone repair by transplanted MSCs.

$5 \quad 3.2$ Induced pluripotent (iPSC) and Embryonic (ESC) stem cells

6 Embryonic stem cells are pluripotent and have the ability to differentiate into all tissue

7 cell types, which consist of ectoderm, endoderm, and mesoderm lineages. Induced pluripotent

8 stem cells, originally developed by Takahashi and coworkers, are cells that have been

9 reprogrammed from terminally differentiated cells, such as skin fibroblasts, into a new

10 pluripotent cell with the ability to self-renew and differentiate into all cell lineages, much like

11 ESCs [70]. These stem cells are versatile and can be derived from individual patients with

12 potential uses in tissue engineering, regenerative medicine, and human disease models. Viability

13 and growth of ESCs and iPSCs in vitro depends on many factors, including the culture substrate,

14 typically a thin-film of Matrigel or a cell feeder layer, and cell morphology and density, typically

15 colonies of cells in two-dimensional culture or three-dimensional cell clusters known as

16 embryoid bodies (EBs) [5]. Researchers are actively designing well-defined dynamic hydrogels

17 for the 3D culture of pluripotent stem cells (PSCs [both ESCs and iPSCs]) to enable their

18 proliferation, expansion, and differentiation, which will be further discussed in this section.

\section{3.2.1 iPSC and ESC proliferation and expansion}

20 The effects of matrix integrin binding, degradability, and crosslink density on ES and iPS

21 cell viability and proliferation have been examined within 4D synthetic matrices toward

22 regulating these processes for stable expansion of pluripotent cells. Hubbell and coworkers

23 screened integrins present on mouse ESCs and subsequently characterized how 
1 microenvironmental factors influenced stem cell renewal and pluripotency in a synthetic

2 hydrogel. Four integrins were identified on these ESCs $\left(\alpha_{5} \beta_{1}, \alpha_{v} \beta_{5}, \alpha_{6} \beta_{1}\right.$, and $\left.\alpha_{9} \beta_{1}\right)$, and to

3 activate them within 3D culture, the synthetic adhesion peptides RGDSP, TTSWSQ, and

4 AEIDGEIL were incorporated within an MMP-degradable (Ac-GCRGD-GPQG $\downarrow$ IWGQ-DRCG-

$5 \mathrm{NH}_{2}$ ) PEG vinyl-sulfone (PEG-VS) hydrogel [71]. This synthetic 4D microenvironment

6 supported ESC pluripotency (OCT4+ marker) and viability for three weeks without passage,

7 similar to ESCs cultured on a cell feeder-layer. With this approach, integrins linked to activation

8 of specific signaling pathways associated with 'stemness' were identified: specifically, Akt1

9 signaling activated by integrin $\alpha_{6} \beta_{1}$ was observed to be critical for stem cell self-renewal.

10 Similarly, Lim and coworkers used MMP-2 degradable 3-, 4-, and 8-arm PEG-VS hydrogels to

11 examine the effect of crosslink density and degradability on the viability and proliferation of

12 three human ESC lines (H1, H9, and Novo hESCs) [72]. Compared to feeder layer 2D culture,

13 culture within the 8-arm PEG-VS degradable matrix resulted in higher proliferation, larger cell

14 cluster size, and increased pluripotency (NANOG, KLF4, SOX2 markers) for H9 ESCs. The 3-

15 arm PEG-VS did not support cell growth, indicating stem cell viability is affected by the

16 mechanical properties or density of the crosslinked hydrogel. Proliferation rates of the three

17 hESC lines were similar in the 8-arm hydrogel, but varied when cultured on feeder-layer 2D

18 substrates, indicating the control of stem cell growth and proliferation afforded by 4D hydrogel-

19 based cultures.

20 Looking at microenvironmental cues more broadly, Lutolf and coworkers recently

21 developed a method that utilizes a nanoliter liquid-dispensing robot to examine the effects of

22 over 1,000 unique 3D microenvironments on regulating self-renewal of ESCs (with a OCT4-

23 GFP reporter) [73]. This microarray platform provided a systems-level understanding of dynamic 
$13 \mathrm{D}$ environments by examining matrix elasticity ( $\mathrm{E} \sim 300$ to $5,400 \mathrm{~Pa}$ ), proteolytic degradability,

2 ECM proteins (Collagen IV, Fibronectin, and Laminin), cell-cell interactions (E-Cadherin,

3 Jagged, and EpCAM), cell density (25 to 200 cells/well), and soluble factors (FGF4, BMP4, and

4 LIF) on cell-matrix interactions. Enzyme-activated transglutaminase factor XIIIa was used to

5 crosslink PEG macromers decorated with reactive peptide sequences to MMP-cleavable peptides

6 with different degradability (Ac-FKGGVPMS $\downarrow$ MRGGERGG-NH ${ }_{2}$, GPQG $\downarrow$ IWGQ, GPQG

$7 \downarrow$ IAGQ, and GDQG $\downarrow$ IAGF, listed with decreasing degradation rate). A generalized linear

8 model was used to quantify and rank factors that significantly affected ESC self-renewal and

9 proliferation. The most influential interactions (from most influential to least) were soluble

10 factors, matrix degradability and stiffness, and tethered proteins and cell density. ESC

11 proliferation was highest when cultured with the soluble factor LIF (leukemia inhibitory factor)

12 in a degradable matrix, and ESC self-renewal was most enhanced when cultured with LIF at a

13 high cell density. Cell-cell interaction proteins (i.e., EpCam) decreased proliferation and self-

14 renewal of encapsulated ESCs, which is in contrast with previously shown support of ESC self-

15 maintenance in $2 \mathrm{D}$ culture. This difference between $2 \mathrm{D}$ and $3 \mathrm{D}$ culture suggests that pathways

16 regulated by cell-cell interaction proteins could be overridden by other factors in dynamic 3D

17 environments. Hydrogels were degraded for downstream cell analyses with qRT-PCR and flow

18 cytometry to assess early germ layer markers. Changes in matrix stiffness resulted in

19 upregulation of ectoderm lineage (Map2). This high-throughput method enabled the

20 identification of a range of microenvironmental properties that are influential for stem cell

21 growth and proliferation.

22 Expansion of human PSCs (hPSCs) can enable large-scale industrial utilization of stem

23 cells in clinical development for biomedical applications. To address the challenge of hPSC 
1 expansion, Schaffer and coworkers developed a fully defined and scalable 4D culture system

2 using a non-covalent thermoreversible hydrogel, $\operatorname{poly}(N$-isopropylacrylamide)-co-poly(ethylene

3 glycol) (PNIPPAAm-PEG) [57,74]. These hydrogels dissolved at lower temperatures, when the

4 polymer network becomes more hydrophilic, and reformed at high temperatures to encapsulate,

5 passage, and expand stem cells. Every passage resulted in $\sim 10$ - or 20 -fold expansion over 4 or 5

6 days, and long-term culture resulted in a cumulative $\sim 10^{72}$-fold expansion with $\sim 95 \%$ of cells

7 remaining pluripotent (Oct4+) at day 280 (Figure 4A). Additionally, EBs cultured in the

8 thermoreversible hydrogels were capable of differentiation into dopaminergic (DA) neurons.

9 These thermoreversible 3D hydrogels supported large-scale expansion of stem cells and

10 ultimately neuronal differentiation.

\section{$11 \quad 3.2 .2$ iPSC and ESC differentiation}

12 The ability of iPSCs and ESCs to differentiate into any cell type makes them attractive

13 for regenerative medicine applications and therapeutic delivery. As observed with regulating

14 proliferation, differentiation of hPSCs in 3D matrices depends on multiple factors, including

15 matrix biomechanical properties, degradability, diffusivity of growth factors, biochemical

16 functionality, and regulation of cell size and shape. An earlier study by Vunjak-Novakovic and

17 coworkers, used HA hydrogels for angiogenic differentiation of encapsulated hESC EBs [75].

18 EBs were differentiated with the addition of VEGF in the culture medium. After one week, cells

19 showed a sprouting morphology and stained positive for the smooth muscle cell marker $\alpha$ -

20 smooth muscle actin, indicating angiogenic differentiation. Dynamic hydrogels also have been

21 used to direct differentiation of size-controlled EBs by recapturing aspects of the

22 microenvironment geometry present during development. Khademhosseini and coworkers

23 encapsulated EBs in RGD-modified MMP-sensitive PEG hydrogels (RGD-PEG) formed by 
1 acrylate chemistry to determine the effect of EB size on endothelial and cardiac differentiation

2 [76]. Microwell arrays within a PEG hydrogel were prepared using a PDMS stamp and seeded

3 with ESCs, controlling the size of EBs formed (either $150 \mu \mathrm{m}$ or $450 \mu \mathrm{m}$ in diameter). After

4 formation, the EBs were collected and encapsulated within the cell-degradable hydrogels. Larger

5 EBs of $450 \mu \mathrm{m}$ encapsulated in RGD-PEG hydrogels differentiated into endothelial cells;

6 however, EBs of $450 \mu \mathrm{m}$ encapsulated without RGD differentiated into cardiomyocytes.

7 Endothelial differentiation was observed with $150 \mu \mathrm{m}$ EBs in both PEG and RGD-PEG

8 hydrogels, but enhanced endothelialization was observed in RGD-PEG hydrogels. Taken

9 together, EB size and matrix functionality influence lineage-specific differentiation.

10 Physical constraints present during embryogenesis also can be emulated by 4D hydrogels.

11 Healy and coworkers used PEG substrates to spatially constrain iPSCs and probe the influence of

12 mechanical stress on their differentiation. Specifically, PEG substrates were stamped to form

13 wells coated only at the bottom with vitronectin or fibronectin, and these wells were seeded with

14 iPSCs. The physical constraint provided by the PEG wells caused cell condensation, a

15 confinement of cells to bias cell migration and ultimately direct differentiation [77]. Cells in the

16 center of the well showed biased migration radially outward, whereas cells on the edge migrated

17 in a random-walk pattern. Additionally, cells at the perimeter differentiated into myofibroblasts,

18 whereas cells at the center differentiated into cardiomyocytes that grew outward and contracted,

19 indicating a beating cardiomyocyte (Figure 4B). This 4D culture resulted in formation of an in

20 vitro cardiac microchamber to mimic human heart development and illustrates how biophysical

21 cues can be utilized for cell lineage differentiation.

22 3.3 Progenitor Cells and Stem Cell-Derived Cells 
Progenitor cells are found in different tissues throughout the body and can differentiate to

2 form one or more cell types in response to microenvironment cues, but unlike stem cells, cannot

3 divide indefinitely. For example, neural progenitor cells (NPCs) are found in the brain and

4 capable of self-renewal and primarily differentiating into neurons, astrocytes, and

5 oligodendrocytes, whereas alveolar type II epithelial cells are found in the alveoli of the lung and

6 capable of self-renewal and differentiation into alveolar type I epithelial cells. Additionally,

$7 \quad$ human stem cells have been differentiated into specific cell types in vitro to provide access to

8 and allow studies of various cell types that otherwise must be acquired invasively (i.e., neuronal

9 subtypes from different regions in the brain). Broadly, progenitor cells and stem cell-derived

10 cells are more specialized but show great promise in regenerative medicine and studies of disease

11 pathology. 4D biomaterials can recapture key features of relevant microenvironments for these

12 cells, supporting cell viability and differentiation and probing of factors that regulate relevant

13 cellular functions, as well as provide a dynamic structure for delivery of these cells in vivo. This

14 section will focus on studies of neural progenitors, cardiac progenitors, and neurons and

15 endothelial cells derived from stem cells.

16 3.3.1 Viability of and neurite outgrowth from NPCs and ESC-derived cells

17 NPCs are some of the most widely studied progenitors owing to their promise for 18 regenerating specific neural tissues and studying neurodegenerative diseases. Dynamic 3D

19 biomaterial-based culture systems have been designed to control relevant mechanical and

20 biochemical properties for mimicking aspects of neural tissue to support NPC viability and

21 differentiation. For example, laminin is a critical structural ECM protein of neural tissue [78];

22 synthetic peptide sequences RGD, YIGSR, and IKVAV have been incorporated within soft,

23 well-defined hydrogel-based matrices to mimic key biological functions of laminin-rich neural 
1 tissue. Segura and coworkers optimized concentrations of these peptides for viability and

2 differentiation of neural progenitor cells derived from iPSCs (iPS-NPCs). iPS-NPCs were 3 encapsulated in 3D MMP-degradable (Ac-CGRDGPQG $\downarrow$ IWGQDRCG-NH 2 ) HA hydrogels

4 decorated with these integrin-binding peptides [79]. IKVAV was observed to have the greatest

5 effect on iPS-NPC viability, and iPS-NPCs subsequently were differentiated into DA neurons

6 within these hydrogels. Compared to differentiation of iPS-NPCs on 2D substrates, the optimized

7 3D hydrogel was beneficial and promoted earlier neuronal differentiation.

8 Neurite extension or axon outgrowth is important in the formation of synapses for neural 9 circuit functionality. Neurite outgrowth from embryonic stem cell-derived motor neurons 10 (ESMNs) in 3D culture was examined by Anseth and coworkers in thiol-ene 4-arm PEG 11 hydrogels functionalized with the peptides CKKKKKK and CYIGSR and crosslinked with an 12 MMP-degradable crosslinker (KCGPQG $\downarrow$ IWGQCK) [80]. The degradable hydrogels supported 13 EB ESMN viability and allowed significantly greater neurite extension, with an average length 14 of $148 \pm 14 \mu \mathrm{m}$, than non-degradable gels. Additionally, a soft modulus $\left(\mathrm{G}^{\prime} \sim 330 \mathrm{~Pa}\right)$ promoted 15 the most motor axon outgrowth. Building upon this to further examine parameters that influence 16 axon extension and the formation of neural circuits, EB ESMNs were co-encapsulated with 17 C2C12 myoblast cells in a photodegradable o-nitrobenzoyl azide (NBA)-functionalized PEG 18 hydrogel formed by a SPAAC reaction [37]. NBA-functionalized hydrogels enabled user control 19 of matrix degradation with single-photon long wavelength UV or two-photon NIR irradiation. 20 Channels were eroded within the matrix to investigate whether biochemical or physical cues 21 influence the path of axon extension. To test the affect of physical constraints on axon extension, 22 pathway options without biochemical cues were presented to extending neurites, and most motor 23 axons were observed to extend toward a $0^{\circ}$ or $45^{\circ}$ direction. Next, using two-photon irradiation, 
1 channels were eroded between encapsulated ESMNs and C2C12s, connecting the two cell types

2 toward the formation of neuromuscular junctions; these junctions are observed in vivo and are

3 usually damaged after a traumatic injury. After two days, synapse formation was observed

4 between ESMNs and $\mathrm{C} 2 \mathrm{C} 12$ s (Figure 4C). This tunable and degradable material permitted

5 neurite extension in three dimensions, coencapsulation, and neural network formation over time

6 and demonstrates the utility of 4D biomaterials for hypothesis testing and directing progenitor

7 cell functions.

\section{$8 \quad$ 3.3.2 Differentiation of neural progenitor cells}

9 As observed with stem cells, NPC differentiation is influenced by biochemical cues, 10 mechanics, and degradability of the surrounding matrix. Werner and coworkers examined the 11 effect of RGD functionality, growth factor sequestration, and hydrogel crosslink density on 12 neuronal differentiation of NPCs. The biohybrid hydrogel was composed of RGD functionalized 13 star-PEG covalently crosslinked with heparin to promote FGF-2 sequestration [81]. The 14 crosslink density was varied by the PEG to heparin ratio to test the effect of modulus on the rate 15 of growth factor diffusion, where $\mathrm{E}$ ranged from $0.18 \pm 0.01 \mathrm{kPa}$ to $4.45 \pm 0.62 \mathrm{kPa}$ correlating 16 with a mesh size of $292 \mathrm{~nm}$ to $103 \mathrm{~nm}$, respectively. The highest crosslink density/modulus 17 hydrogel reduced cell viability; however, crosslink density did not influence FGF-2 sequestration 18 due to the strong affinity of FGF-2 for heparin. Hydrogels functionalized with RGD and heparin

19 resulted in increased differentiation of NPCs into neurons when compared to non-functionalized

20 hydrogels. These results indicate that matrix biofunctionality and growth factor sequestration

21 play a more significant role in neural differentiation than modulus of these materials within the 22 property ranges examined. 
In a parallel study, Schmidt and coworkers showed how the degradability of

2 methacrylated HA (MAHA) hydrogels promoted NPC migration and differentiation into neurons

3 [82]. The compressive modulus of these hydrogels was varied from $1.5 \pm 0.03 \mathrm{kPa}$ to $7.2 \pm 0.03$

$4 \mathrm{kPa}$. The softest hydrogels promoted NPC differentiation into dopaminergic neurons, and the

5 stiffest hydrogels promoted NPC differentiation into astrocytes. Migration of NPCs within these

6 hydrogels was inhibited by the highly crosslinked networks and their corresponding small mesh

7 size that restricted cell movement. Based on these observations, the authors hypothesized that

8 differentiating NPCs first extend neurite processes and then migrate through the hydrogel as the

9 hydrogel degrades and the mesh size increases over time. These results suggest that promoting

10 neurite extension in soft moduli hydrogels can be utilized to enhance neuronal differentiation.

11 Commonalities and differences in the findings of these two examples illustrate how the structure

12 and biochemical content of biomimetic microenvironments influence NPC differentiation. More

13 broadly, further studies such as these using 4D biomaterials may aid in determining the influence

14 of different microenvironmental factors on the differentiation of other types of progenitor cells.

$15 \quad$ 3.3.3 Delivery of cells in vivo

16 Maintaining viability of cells after delivery in vivo is a current issue for regenerative

17 medicine therapies. Dynamic hydrogels have improved cell survival and differentiation after in

18 vivo transplantation by protecting them from damage during injection through syringe needles.

19 For example, using the MITCH assembly strategy (see section 2), Heilshorn and coworkers

20 formed hybrid hydrogels for 3D cell culture using multi-armed PEG conjugated with proline-rich

21 peptides and proteins containing C7 and RGD domains [83]. These MITCH-PEG hydrogels

22 exhibit thixotrophy, or the ability to shear-thin and self-heal. The authors hypothesized

23 thixotrophic hydrogels could increase cell viability for iPSC-derived endothelial cells (hiPSC- 
1 ECs) during syringe injection. Additionally, vascular endothelial growth factor (VEGF) was

2 added to the gel-forming mixture, and crosslink density and binding domains were varied to

3 control the rate of gel erosion and VEGF release after injection. These hydrogels were observed

4 to protect cells from damage during injection (i.e., increased cell viability relative to controls)

5 and promoted muscle tissue regeneration with the co-delivery of hiPSC-ECs and VEGF in vivo.

6 Healy and coworkers have used Matrix Assisted Cell Transplantation (MACT) hydrogels

7 to evaluate adhesive peptide functionality, mechanical properties, and growth factor

8 sequestration for survival, differentiation, and capillary tube formation of cardiac progenitor cells

9 (CPCs) after transplantation [84]. Acrylated HA was reacted by Michael-type addition with a

10 bis-cysteine MMP-degradable crosslinker (CQPQGLAKC) and different combinations of thiol-

11 modified RGD or heparin (HyA-PHT hydrogel). With a high affinity for heparin, TGF $\beta 1$ was

12 added to the gel-forming solution to promote endothelial differentiation. CPCs cultured in HyA-

13 PHT gels differentiated into endothelial cells and formed of a tubular network in vitro and in vivo

14 upon transplantation, with the largest network forming in high moduli gels (850 Pa) with an

15 RGD density of $380 \mu \mathrm{M}$. These results support the promise of 4D hydrogel-based materials for

16 both in vitro differentiation and in vivo delivery of progenitor cells.

\section{4. Conclusion}

4D hydrogel-based biomaterials permit in-depth studies of complex stem cell processes.

20 With a variety of chemistries including covalent addition, non-covalent interactions, and

21 degradation, a wide range of dynamic materials have been constructed for the culture of cells in

22 three dimensions. These biocompatible materials allow controlled studies of how matrix

23 properties regulate stem cell viability, proliferation, and differentiation and enable in vitro 
1 studies of specific phenomena during morphogenesis. Additionally, these materials are

2 promising for regenerative medicine applications by improving transplantation methods for the

3 co-delivery of cells and growth factors to sights of injury. As research progresses to examine

4 more specific biological processes, 4D biomaterials provide a platform for stem cell-derived in

$5 \quad$ vitro model systems to study specific processes during regeneration or disease progression.

6

\section{Acknowledgements}

8 The authors would like to acknowledge support, for related work in their laboratories,

9 from the National Science Foundation CAREER Award (DMR-1253906), the Delaware COBRE

10 program with a grant from the National Institute of General Medical Sciences (NIGMS

11 P20GM104316) from the NIH, the NIH Chemistry-Biology Interface program at the University

12 of Delaware (NIH T32GM008550), the Burroughs Wellcome Fund, and the Pew Charitable

13 Trusts (00026178).

14

15

16

17 
References

2 [1] National Institues of Health USD of H and HS. Stem Cell Basics. Stem Cell Inf 2009.

[8] Sahoo S, Chung C, Khetan S, Burdick JA. Hydrolytically degradable hyaluronic acid hydrogels with controlled temporal structures. Biomacromolecules 2008;9:1088-92. doi:10.1021/bm800051m.

[9] Feng Q, Zhu M, Wei K, Bian L. Cell-Mediated Degradation Regulates Human Mesenchymal Stem Cell Chondrogenesis and Hypertrophy in MMP-Sensitive Hyaluronic Acid Hydrogels. PLoS One 2014;9:e99587.

[10] Murphy WL, McDevitt TC, Engler AJ. Materials as stem cell regulators. Nat Mater 2014;13:547-57. doi:10.1038/nmat3937.

[11] Weber LM, Lopez CG, Anseth KS. Effects of PEG hydrogel crosslinking density on protein diffusion and encapsulated islet survival and function. J Biomed Mater Res Part A 2009;90A:720-9.

[12] McCall JD, Lin C-C, Anseth KS. Affinity peptides protect transforming growth factor beta during encapsulation in poly(ethylene glycol) hydrogels. Biomacromolecules 2011;12:1051-7.

[13] Agard NJ, Prescher JA, Bertozzi CR. A strain-promoted [3 + 2] azide-alkyne cycloaddition for covalent modification of biomolecules in living systems. J Am Chem Soc 2004;126:15046-7. doi:10.1021/ja044996f.

[14] Xi W, Scott TF, Kloxin CJ, Bowman CN. Click Chemistry in Materials Science. Adv Funct Mater 2014;24:2572-90. doi:10.1002/adfm.201302847.

[15] DeForest CA, Polizzotti BD, Anseth KS. Sequential click reactions for synthesizing and 
patterning three-dimensional cell microenvironments. Nat Mater 2009;8:659-64. doi:10.1038/nmat2473.

[16] Zheng J, Smith Callahan LA, Hao J, Guo K, Wesdemiotis C, Weiss RA, et al. StrainPromoted Crosslinking of PEG-based Hydrogels via Copper-Free Cycloaddition. ACS Macro Lett 2012;1:1071-3.

[17] Hoyle CE, Bowman CN. Thiol-ene click chemistry. Angew Chem Int Ed Engl 2010;49:1540-73. doi:10.1002/anie.200903924.

[18] Gramlich WM, Kim IL, Burdick JA. Synthesis and orthogonal photopatterning of hyaluronic acid hydrogels with thiol-norbornene chemistry. Biomaterials 2013;34:980311.

[19] Fairbanks BD, Sims EA, Anseth KS, Bowman CN. Reaction Rates and Mechanisms for Radical, Photoinitated Addition of Thiols to Alkynes, and Implications for Thiol-Yne Photopolymerizations and Click Reactions. Macromolecules 2010;43:4113-9. doi:10.1021/ma1002968.

[20] Truong VX, Ablett MP, Richardson SM, Hoyland JA, Dove AP. Simultaneous orthogonal dual-click approach to tough, in-situ-forming hydrogels for cell encapsulation. J Am Chem Soc 2015;137:1618-22. doi:10.1021/ja511681s.

[21] Nimmo CM, Owen SC, Shoichet MS. Diels-Alder Click cross-linked hyaluronic acid hydrogels for tissue engineering. Biomacromolecules 2011;12:824-30.

[22] Yu F, Cao X, Li Y, Chen X. Diels-Alder Click-Based Hydrogels for Direct Spatiotemporal Postpatterning via Photoclick Chemistry. ACS Macro Lett 2015;4:289-92. doi:10.1021/mz5007427.

[23] Grover GN, Lam J, Nguyen TH, Segura T, Maynard HD. Biocompatible hydrogels by oxime Click chemistry. Biomacromolecules 2012;13:3013-7. doi:10.1021/bm301346e.

[24] Lin F, Yu J, Tang W, Zheng J, Defante A, Guo K, et al. Peptide-functionalized oxime hydrogels with tunable mechanical properties and gelation behavior. Biomacromolecules 2013;14:3749-58. doi:10.1021/bm401133r.

[25] Kharkar PM, Kiick KL, Kloxin AM. Designing degradable hydrogels for orthogonal control of cell microenvironments. Chem Soc Rev 2013;42:7335-72.

[26] Buwalda SJ, Dijkstra PJ, Calucci L, Forte C, Feijen J. Influence of amide versus ester linkages on the properties of eight-armed PEG-PLA star block copolymer hydrogels. Biomacromolecules 2010;11:224-32. doi:10.1021/bm901080d.

[27] Hao Y, Shih H, Muňoz Z, Kemp A, Lin C-C. Visible light cured thiol-vinyl hydrogels with tunable degradation for 3D cell culture. Acta Biomater 2014;10:104-14.

[28] Lévesque SG, Shoichet MS. Synthesis of enzyme-degradable, peptide-cross-linked dextran hydrogels. Bioconjug Chem 2007;18:874-85.

[29] Lutolf MP, Hubbell JA. Synthetic biomaterials as instructive extracellular microenvironments for morphogenesis in tissue engineering. Nat Biotechnol 2005;23:4755. doi:10.1038/nbt1055.

[30] Chung C, Beecham M, Mauck RL, Burdick JA. The influence of degradation characteristics of hyaluronic acid hydrogels on in vitro neocartilage formation by 
[31] Khetan S, Guvendiren M, Legant WR, Cohen DM, Chen CS, Burdick JA. Degradationmediated cellular traction directs stem cell fate in covalently crosslinked threedimensional hydrogels. Nat Mater 2013;12:458-65.

[32] Kloxin AM, Kasko AM, Salinas CN, Anseth KS. Photodegradable hydrogels for dynamic tuning of physical and chemical properties. Science (80- ) 2009;324:59-63.

[33] Lee MS, Kim JC. Photodependent release from poly(vinyl alcohol)/epoxypropoxy coumarin hydrogels. J Appl Polym Sci 2012;124:4339-45. doi:10.1002/app.35411.

[34] Azagarsamy MA, McKinnon DD, Alge DL, Anseth KS. Coumarin-Based Photodegradable Hydrogel: Design, Synthesis, Gelation, and Degradation Kinetics. ACS Macro Lett 2014;3:515-9.

[35] Griffin DR, Patterson JT, Kasko AM. Photodegradation as a mechanism for controlled drug delivery. Biotechnol Bioeng 2010;107:1012-9.

[36] Griffin DR, Kasko AM. Photodegradable macromers and hydrogels for live cell encapsulation and release. J Am Chem Soc 2012;134:13103-7.

[37] McKinnon DD, Brown TE, Kyburz KA, Kiyotake E, Anseth KS. Design and characterization of a synthetically accessible, photodegradable hydrogel for user-directed formation of neural networks. Biomacromolecules 2014;15:2808-16.

[38] DeForest CA, Anseth KS. Cytocompatible click-based hydrogels with dynamically tunable properties through orthogonal photoconjugation and photocleavage reactions. Nat Chem 2011;3:925-31.

[39] Zhu C, Bettinger CJ. Light-induced remodeling of physically crosslinked hydrogels using near-IR wavelengths. J Mater Chem B 2014;2:1613-8.

[40] Lux C de G, Lux J, Collet G, He S, Chan M, Olejniczak J, et al. Short Soluble Coumarin Crosslinkers for Light-Controlled Release of Cells and Proteins from Hydrogels 2015.

[41] Stowers RS, Allen SC, Suggs LJ. Dynamic phototuning of 3D hydrogel stiffness. Proc Natl Acad Sci U S A 2015:1421897112 - . doi:10.1073/pnas.1421897112.

[42] Choh SY, Cross D, Wang C. Facile synthesis and characterization of disulfide-crosslinked hyaluronic acid hydrogels for protein delivery and cell encapsulation. Biomacromolecules 2011;12:1126-36. doi:10.1021/bm101451k.

[43] Koehler KC, Alge DL, Anseth KS, Bowman CN. A Diels-Alder modulated approach to control and sustain the release of dexamethasone and induce osteogenic differentiation of human mesenchymal stem cells. Biomaterials 2013;34:4150-8.

[44] Baldwin AD, Kiick KL. Tunable degradation of maleimide-thiol adducts in reducing environments. Bioconjug Chem 2011;22:1946-53.

[45] Kharkar PM, Kloxin AM, Kiick KL. Dually degradable click hydrogels for controlled degradation and protein release. J Mater Chem B 2014. doi:10.1039/C4TB00496E.

[46] Kharkar PM, Kiick KL, Kloxin AM. Design of thiol-and light-sensitive degradable hydrogels using Michael-type addition reactions. Polym Chem 2015.

[47] Rosales AM, Mabry KM, Nehls EM, Anseth KS. Photoresponsive elastic properties of 
azobenzene-containing poly(ethylene-glycol)-based hydrogels. Biomacromolecules 2015;16:798-806. doi:10.1021/bm501710e.

[48] Altunbas A, Lee SJ, Rajasekaran S a., Schneider JP, Pochan DJ. Encapsulation of curcumin in self-assembling peptide hydrogels as injectable drug delivery vehicles. Biomaterials 2011;32:5906-14. doi:10.1016/j.biomaterials.2011.04.069.

[49] Banwell EF, Abelardo ES, Adams DJ, Birchall MA, Corrigan A, Donald AM, et al. Rational design and application of responsive alpha-helical peptide hydrogels. Nat Mater 2009;8:596-600. doi:10.1038/nmat2479.

[50] Greenwood-Goodwin M, Teasley ES, Heilshorn SC. Dual-stage growth factor release within 3D protein-engineered hydrogel niches promotes adipogenesis. Biomater Sci 2014;2:1627-39. doi:10.1039/C4BM00142G.

[51] Huang CC, Ravindran S, Yin Z, George A. 3-D self-assembling leucine zipper hydrogel with tunable properties for tissue engineering. Biomaterials 2014;35:5316-26. doi:10.1016/j.biomaterials.2014.03.035.

[52] Pérez CMR, Panitch A, Chmielewski J. A collagen peptide-based physical hydrogel for cell encapsulation. Macromol Biosci 2011;11:1426-31.

[53] Scott CM, Forster CL, Kokkoli E. 3D Cell Entrapment as a Function of the Weight Percent of Peptide-Amphiphile Hydrogels. Langmuir 2015:150513155506007. doi:10.1021/acs.langmuir.5b00196.

[54] Silva G a, Czeisler C, Niece KL, Beniash E, Harrington D a, Kessler J a, et al. Selective differentiation of neural progenitor cells by high-epitope density nanofibers. Science 2004;303:1352-5. doi:10.1126/science.1093783.

[55] Jayawarna V, Ali M, Jowitt T a., Miller a. F, Saiani a., Gough JE, et al. Nanostructured Hydrogels for Three-Dimensional Cell Culture Through Self-Assembly of Fluorenylmethoxycarbonyl-Dipeptides. Adv Mater 2006;18:611-4. doi:10.1002/adma.200501522.

[56] Zhang Z, Lai Y, Yu L, Ding J. Effects of immobilizing sites of RGD peptides in amphiphilic block copolymers on efficacy of cell adhesion. Biomaterials 2010;31:787382. doi:10.1016/j.biomaterials.2010.07.014.

[57] Lei Y, Schaffer D V. A fully defined and scalable 3D culture system for human pluripotent stem cell expansion and differentiation. Proc Natl Acad Sci U S A 2013;110:E5039-48. doi:10.1073/pnas.1309408110.

[58] Nombela-Arrieta C, Ritz J, Silberstein LE. The elusive nature and function of mesenchymal stem cells. Nat Rev Mol Cell Biol 2011;12:126-31. doi:10.1038/nrm3049.

[59] Ren G, Chen X, Dong F, Li W, Ren X, Zhang Y, et al. Concise review: mesenchymal stem cells and translational medicine: emerging issues. Stem Cells Transl Med 2012;1:518. doi:10.5966/sctm.2011-0019.

[60] Rahmany MB, Van Dyke M. Biomimetic approaches to modulate cellular adhesion in biomaterials: A review. Acta Biomater 2013;9:5431-7. doi:10.1016/j.actbio.2012.11.019.

[61] Anderson SB, Lin C-C, Kuntzler D V, Anseth KS. The performance of human mesenchymal stem cells encapsulated in cell-degradable polymer-peptide hydrogels. 
[62] DeForest CA, Tirrell DA. A photoreversible protein-patterning approach for guiding stem cell fate in three-dimensional gels. Nat Mater 2015;14:523-31. doi:10.1038/nmat4219.

[63] Bahney CS, Hsu C-W, Yoo JU, West JL, Johnstone B. A bioresponsive hydrogel tuned to chondrogenesis of human mesenchymal stem cells. FASEB J 2011;25:1486-96. doi:10.1096/fj.10-165514.

[64] Solorio LD, Dhami CD, Dang PN, Vieregge EL, Alsberg E. Spatiotemporal regulation of chondrogenic differentiation with controlled delivery of transforming growth factor- $\beta 1$ from gelatin microspheres in mesenchymal stem cell aggregates. Stem Cells Transl Med 2012;1:632-9. doi:10.5966/sctm.2012-0039.

[65] Strioga M, Viswanathan S, Darinskas A, Slaby O, Michalek J. Same or not the same? Comparison of adipose tissue-derived versus bone marrow-derived mesenchymal stem and stromal cells. Stem Cells Dev 2012;21:2724-52. doi:10.1089/scd.2011.0722.

[66] Chamberlain G, Fox J, Ashton B, Middleton J. Concise review: mesenchymal stem cells: their phenotype, differentiation capacity, immunological features, and potential for homing. Stem Cells 2007;25:2739-49. doi:10.1634/stemcells.2007-0197.

[67] Mosiewicz K a., Kolb L, van der Vlies AJ, Martino MM, Lienemann PS, Hubbell J a., et al. In situ cell manipulation through enzymatic hydrogel photopatterning. Nat Mater 2013;12:1072-8. doi:10.1038/nmat3766.

[68] Schultz KM, Kyburz K a, Anseth KS. Measuring dynamic cell-material interactions and remodeling during 3D human mesenchymal stem cell migration in hydrogels. Proc Natl Acad Sci U S A 2015;112:E3757-64. doi:10.1073/pnas.1511304112.

[69] Huebsch N, Lippens E, Lee K, Mehta M, Koshy ST, Darnell MC, et al. Matrix Elasticity of Void-Forming Hydrogels Controls Matrix elasticity of void-forming hydrogels controls Transplanted Stem Cell-Mediated bone 2015:1-19. doi:10.1038/NMAT4407.

[70] Takahashi K, Tanabe K, Ohnuki M, Narita M, Ichisaka T, Tomoda K, et al. Induction of pluripotent stem cells from adult human fibroblasts by defined factors. Cell 2007;131:861-72. doi:10.1016/j.cell.2007.11.019.

[71] Lee ST, Yun JI, Jo YS, Mochizuki M, van der Vlies AJ, Kontos S, et al. Engineering integrin signaling for promoting embryonic stem cell self-renewal in a precisely defined niche. Biomaterials 2010;31:1219-26. doi:10.1016/j.biomaterials.2009.10.054.

[72] Jang M, Lee ST, Kim JW, Yang JH, Yoon JK, Park JC, et al. A feeder-free, defined threedimensional polyethylene glycol-based extracellular matrix niche for culture of human embryonic stem cells. Biomaterials 2013;34:3571-80. doi:10.1016/j.biomaterials.2013.01.073.

[73] Ranga A, Gobaa S, Okawa Y, Mosiewicz K, Negro A, Lutolf MP. 3D niche microarrays for systems-level analyses of cell fate. Nat Commun 2014;5:1-10. doi:10.1038/ncomms5324.

[74] McDevitt TC. Scalable culture of human pluripotent stem cells in 3D. Proc Natl Acad Sci U S A 2013;110:20852-3.

41 [75] Gerecht S, Burdick J a, Ferreira LS, Townsend S a, Langer R, Vunjak-Novakovic G. 
Hyaluronic acid hydrogel for controlled self-renewal and differentiation of human embryonic stem cells. Proc Natl Acad Sci U S A 2007;104:11298-303. doi:10.1073/pnas.0703723104.

[76] Schukur L, Zorlutuna P, Cha JM, Bae H, Khademhosseini A. Directed differentiation of size-controlled embryoid bodies towards endothelial and cardiac lineages in RGDmodified poly(ethylene glycol) hydrogels. Adv Healthc Mater 2013;2:195-205. doi:10.1002/adhm.201200194.

[77] Ma Z, Wang J, Loskill P, Huebsch N, Koo S, Svedlund FL, et al. Self-organizing human cardiac microchambers mediated by geometric confinement. Nat Commun 2015;6:7413. doi:10.1038/ncomms8413.

[78] Yamada Y, Hozumi K, Nomizu M. Construction and activity of a synthetic basement membrane with active laminin peptides and polysaccharides. Chem - A Eur J 2011;17:10500-8. doi:10.1002/chem.201101064.

[79] Lam J, Carmichael ST, Lowry WE, Segura T. Hydrogel Design of Experiments Methodology to Optimize Hydrogel for iPSC-NPC Culture. Adv Healthc Mater 2014:n/a - n/a. doi:10.1002/adhm.201400410.

[80] McKinnon DD, Kloxin AM, Anseth KS. Synthetic hydrogel platform for threedimensional culture of embryonic stem cell-derived motor neurons. Biomater Sci 2013;1:460. doi:10.1039/c3bm00166k.

[81] Freudenberg U, Hermann A, Welzel PB, Stirl K, Schwarz SC, Grimmer M, et al. A starPEG-heparin hydrogel platform to aid cell replacement therapies for neurodegenerative diseases. Biomaterials 2009;30:5049-60. doi:10.1016/j.biomaterials.2009.06.002.

[82] Seidlits SK, Khaing ZZ, Petersen RR, Nickels JD, Vanscoy JE, Shear JB, et al. The effects of hyaluronic acid hydrogels with tunable mechanical properties on neural progenitor cell differentiation. Biomaterials 2010;31:3930-40. doi:10.1016/j.biomaterials.2010.01.125.

[83] Mulyasasmita W, Cai L, Dewi RE, Jha A, Ullmann SD, Luong RH, et al. Aviditycontrolled hydrogels for injectable co-delivery of induced pluripotent stem cell-derived endothelial cells and growth factors. J Control Release 2014. doi:10.1016/j.jconrel.2014.05.015.

[84] Jha AK, Tharp KM, Ye J, Santiago-Ortiz JL, Jackson WM, Stahl A, et al. Enhanced survival and engraftment of transplanted stem cells using growth factor sequestering hydrogels. Biomaterials 2015;47:1-12. doi:10.1016/j.biomaterials.2014.12.043. 


\section{Figure Captions}

Figure 1. Changes in stem cell microenvironments in vivo captured by 4D biomaterials in vitro.

A) The microenvironment of stem cells in the body changes with time. These changes have been observed to modulate cellular fate and functions, such as $i$ ) migration in response to gradients of matrix density/stiffness, $i$ ) proliferation in response to matrix remodeling, and iii) differentiation in response to soluble factors (e.g., growth factors). B) Creating materials that capture such changes aids in the study of how cells respond to microenvironment remodeling events, such as wound healing or disease progression, toward ultimately directing these processes. For example, 4D hydrogel-based biomaterials have been engineered to enable i) changes in the mechanical properties of synthetic matrices by the addition or removal of crosslinks, influencing cell migration throughout the materials. At higher crosslink densities and moduli, cells have been entrapped within hydrogel-based matrices (left), whereas at lower crosslink densities and moduli cell migration has been observed (right). ii) Variation in biochemical content within the hydrogels through addition or removal of biochemical moieties (e.g., integrin-binding peptides or protein fragments) has been observed to promote cell proliferation. iii) Addition or sequestration of growth factors swollen within or tethered to the synthetic matrix has been observed to regulate stem cell differentiation. Note, these examples are meant to be representative, rather than comprehensive, of the ways 4D biomaterials have and can be used to probe stem cell processes; many extracellular cues influence multiple cellular functions (e.g., growth factors can influence migration, proliferation, and differentiation).

Figure 2. Chemistries to form and modify hydrogels for 4D culture of stem cells. A) 'Click' reactions, which occur under mild conditions and proceed close to $100 \%$ conversion, often have been used to form and modify hydrogels-based biomaterials in the presence of stem cells. These reactions have been used individually or in combination with each other. Here, a representative sample of the different functional groups and their reactions that have been used for hydrogel formation or modification in the presence of cells are noted: $i$ ) SPAAC, ii) thiol-ene and thiolyne, iii) Diels-Alder cycloaddition, and iv) oxime ligation. B) Further, degradation or cleavage reactions have been used to change the properties of hydrogels during culture through the removal of crosslinks or pendant groups with $i$ ) preprogrammed hydrolysis, ii) cell-driven enzymatic hydrolysis, or ii) externally-triggered photolysis. C) Non-covalent interactions also have been used to form assembled hydrogels for use as dynamic stem cell culture matrices: $i$ ) ionic interactions, ii) hydrophobicity, and iii) hydrogen bonding.

Figure 3. 4D biomaterials for MSC culture. A) Photoreversible patterning of the protein vitronectin within well-defined hydrogel-based matrices controls encapsulated MSC differentiation. PEG-based hydrogels were formed by strain promoted azide-alkyne cycloaddition and B) patterned with vitronectin (dashed line regions), which promoted osteogenic differentiation (green stain indicates cells expressing the osteogenic marker osteocalcin). The regions of the hydrogel with no vitronectin exhibit limited MSC interaction with the matrix (e.g., rounded cell morphology) and limited osteogenic differentiation (no green), where all of the MSCs were stained with CellTracker Red. Adapted from DeForest and Tirrell with permission from Nature Publishing Group [62]. C) MMP-degradable HA hydrogels that allow MSC spreading and traction force generation (-) promote osteogenic differentiation. After 
secondary crosslinking at day 7 (D7 UV), spread cells are unable to degrade the matrix, affecting their ability to generate traction forces, and D) promoting differentiation into adipocytes instead of osteoblasts. Adapted from Burdick and coworkers with permission from Nature Publishing Group [31].

Figure 4. Stem and progenitor cell processes in 4D biomaterials. A) Long term expansion of hPSCs has been achieved in thermoreversible PNIPPAAm-PEG hydrogels. Seven hPSC lines were cultured for multiple passages within these materials with $\sim 95 \%$ pluripotency, indicated by Oct4 expression. The longest hPSC line passage accumulated to $\sim 10^{72}$-fold expansion at 60 passages or 280 days. Adapted from Lei and Schaffer with permission from Proceedings of the National Academy of Sciences USA PNAS [57]. B) 3D cardiac microchamber was formed by confinement of iPSCs within PEG microwells. Cell-laden PEG microwells (top) were fabricated by PEG polymerization, PDMS mask and etching of the PEG film, coating of wells, and seeding of cells. Cells (bottom, nuclei blue) in the center differentiated into cardiomyocytes, as indicated by sarcomeric $\alpha$-actinin stain (red). Cells along the perimeter differentiated into myofibroblasts, as indicated by calponin stain (green). $x-z$ and $y-z$ cross section projections (above and to the left) show the inner void space, indicating a 3D cardiac microchamber. Adapted from Healy and coworkers with permission from Nature Communications Publishing Group [77]. C) EB ESMNs were co-encapsulated with $\mathrm{C} 2 \mathrm{C} 12$ cells within a photodegradable hydrogel. Channels $(10 \mu \mathrm{m}$ $\times 10 \mu \mathrm{m}$ ) were degraded between these two cell types using a 740-nm two-photon laser to cleave the $o$-nitrobenzyl-containing crosslinker (left). At two days, synapses (stained with alphabungarotoxin, red) were observed between motor axon extensions (green) and myotubes (right). Adapted from Anseth and coworkers with permission from Biomacromolecules the American 24 Chemical SocietyACS Publications [37]. 


\section{A. Changes in stem cell microenvironment in vivo}
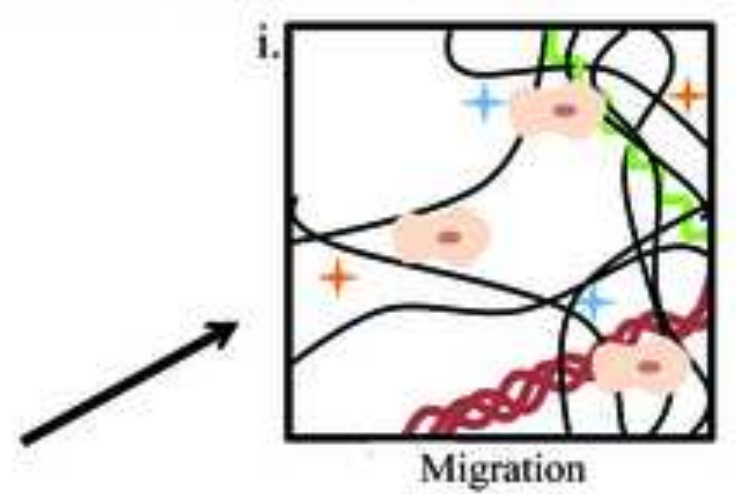

Initial environment
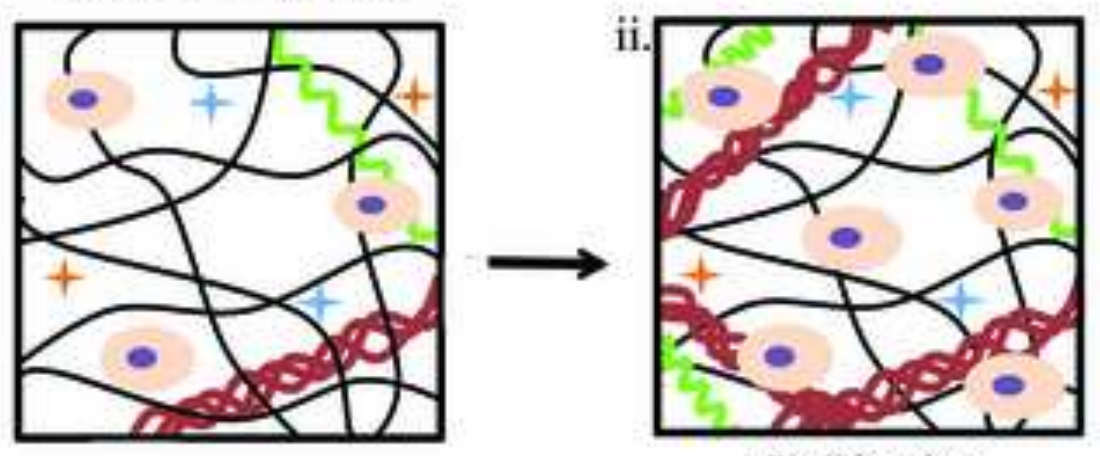

Proliferation

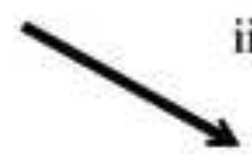

ECM proteins collagen

++ Soluble factors - growth factors

$\int$ Peptide mimics of ECM proteins
Differentiation

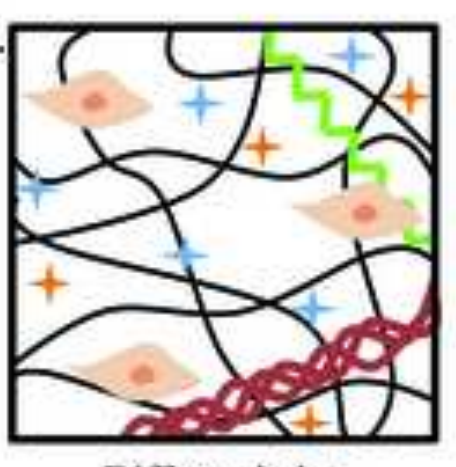

\section{B. 4D biomaterials to mimic dynamic in vivo stem cell} microenvironments

i. Stiff environment Soft environment
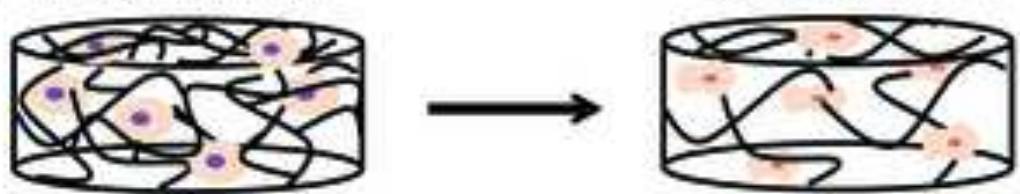

Changes in mechanical properties can influence cell migration

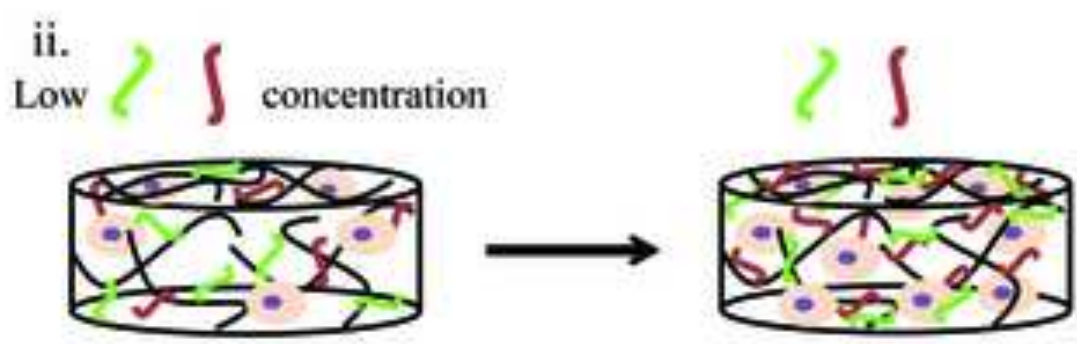

Changes in peptide content can promote cell proliferation

iii.

Low ++ concentration $\quad$ High ++ concentration

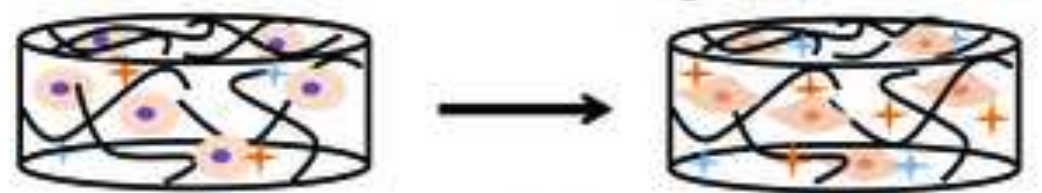

Addition of growth factors can lead to stem cell differentiation 


\section{A. Addition Reactions}

i. Strain promoted azide-alkyne cycloaddition (SPAAC)

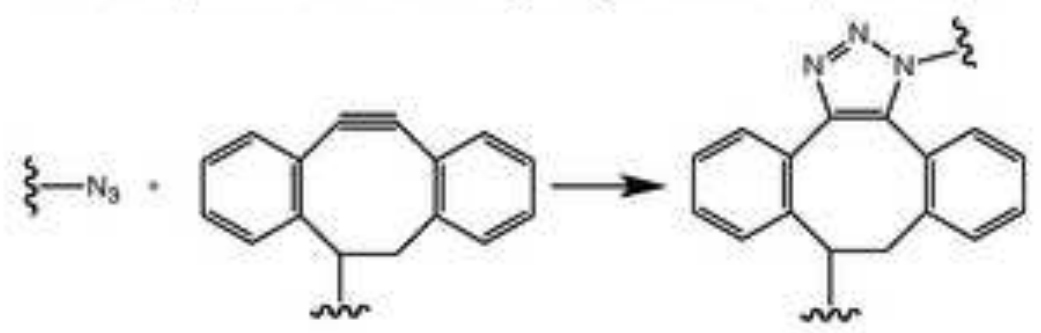

ii. Thiol-ene and thiol-yne

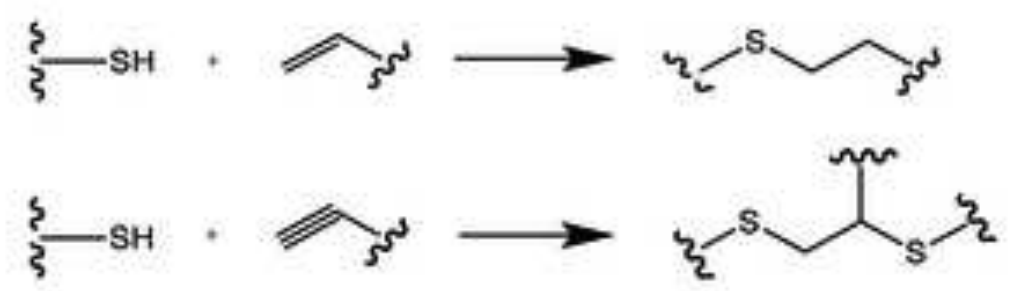

iii. Diels-Alder cycloaddition

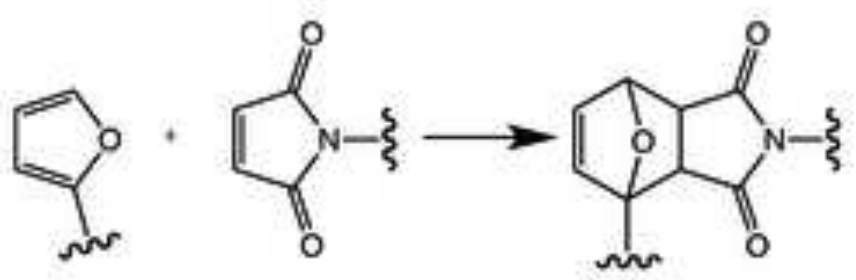

iv. Oxime Ligation

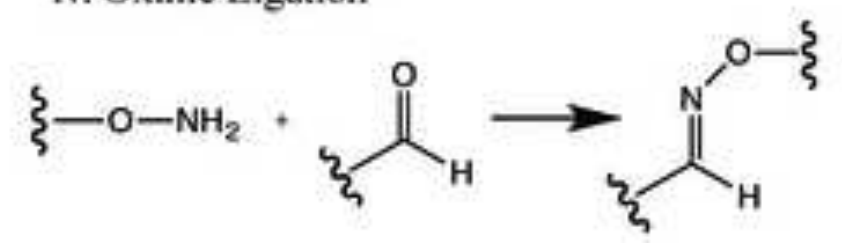

\section{B. Cleavage Reactions}

i. Ester hydrolysis

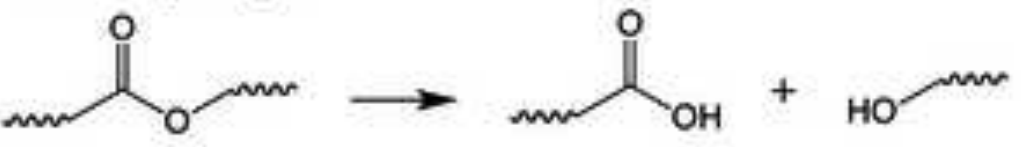

ii. Enzymatic cleavage

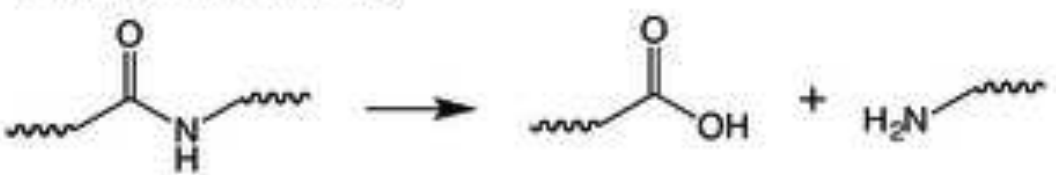

iii. Photodegradation
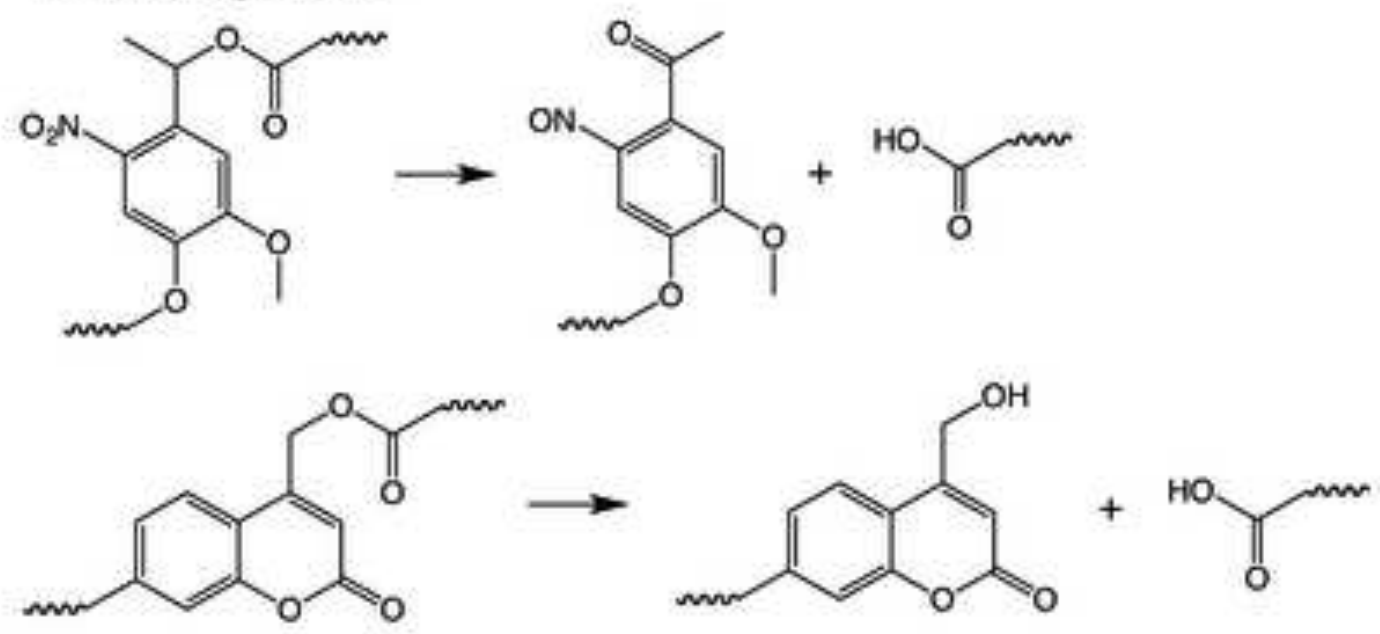

\section{Non-covalent interactions}

i. Ionic interactions

ii. Hydrophobic interactions
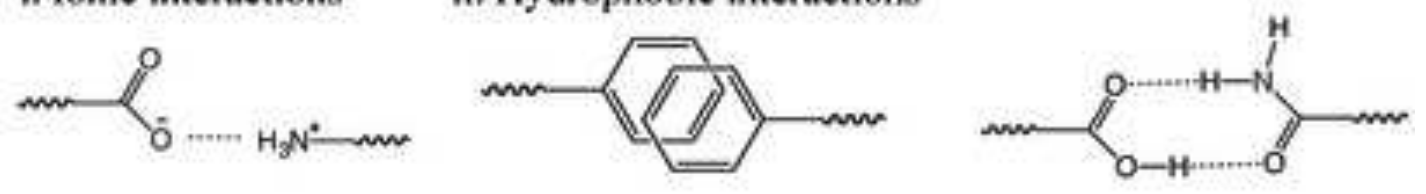
A<smiles>[R]N=[N+]=[N-]</smiles>

C

\section{B}

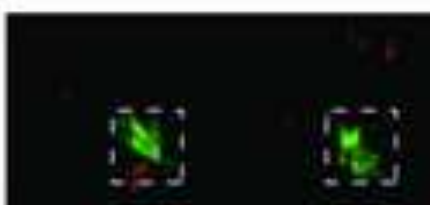

S
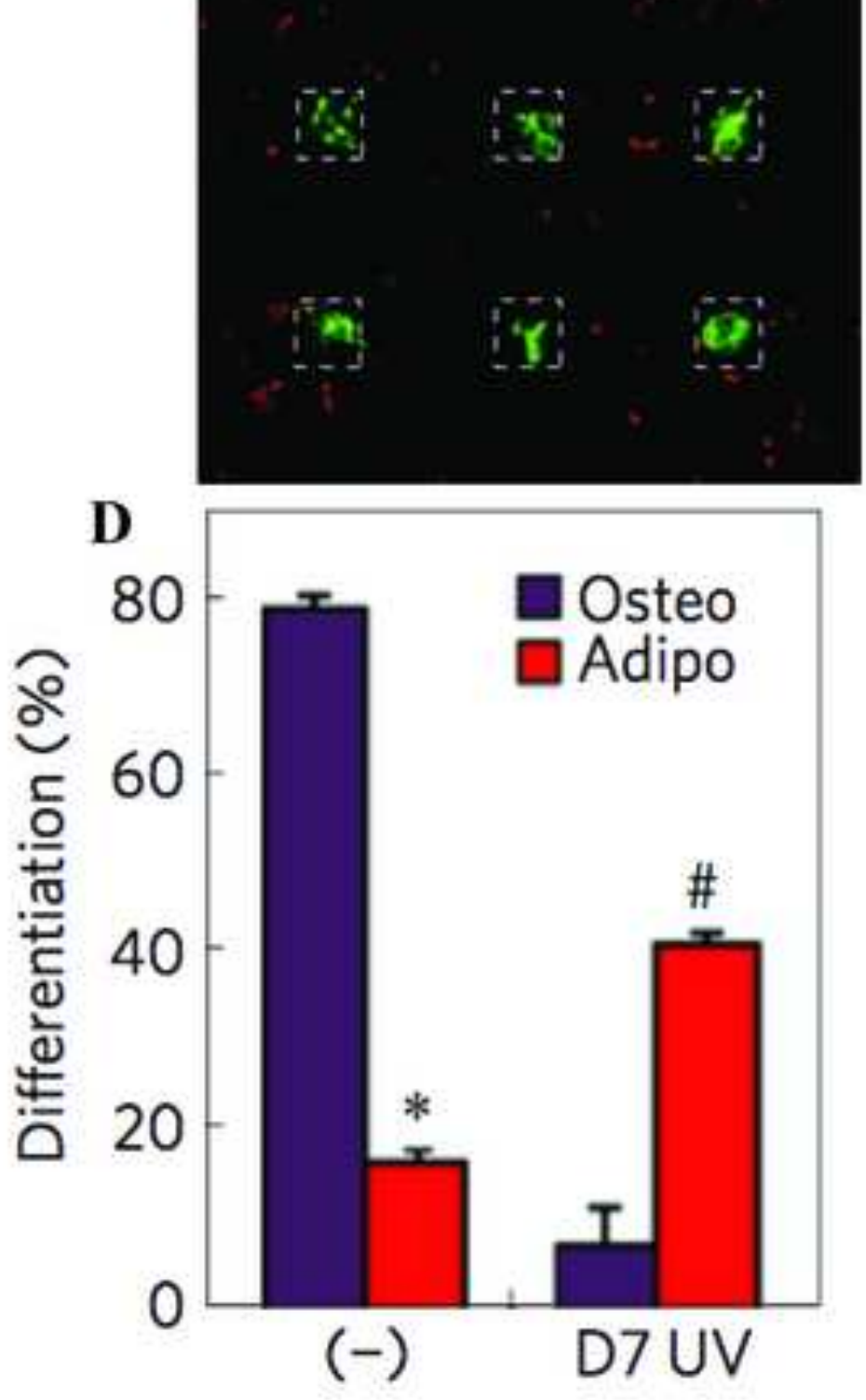
A $"$ H1, Single
"H9, Precluster

"iPS-Fib2, Single

$=H 9$. Single

-iPS-MSC, Precluster $=\mathrm{H} 1$, Precluster

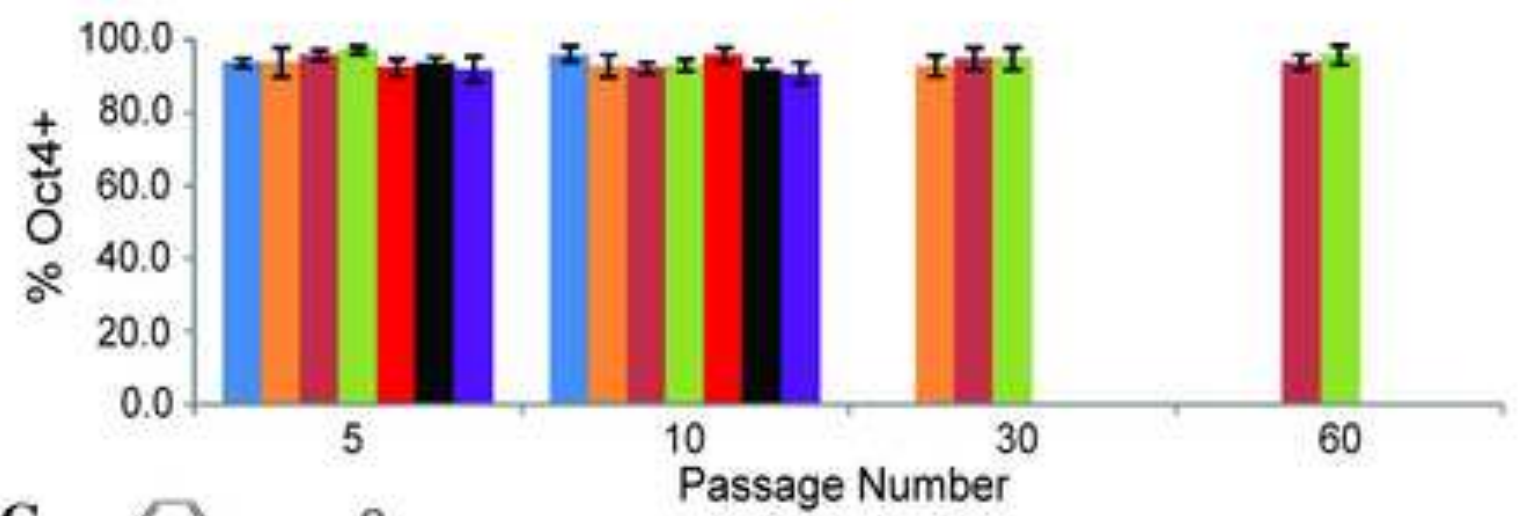

C

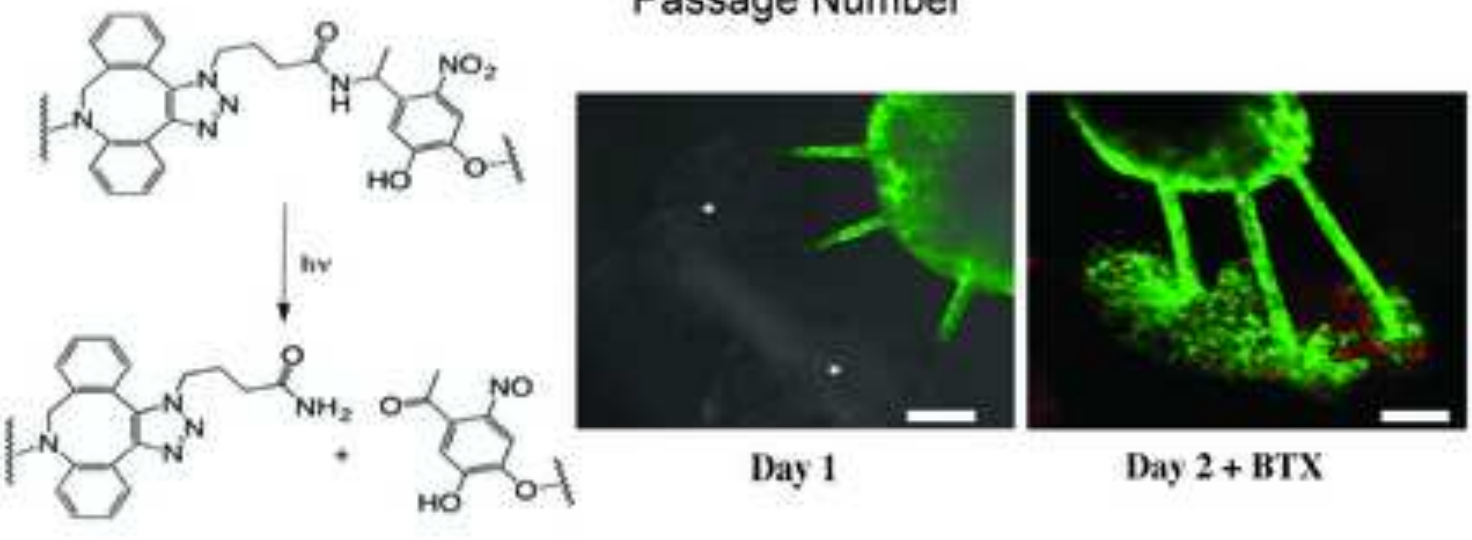

B
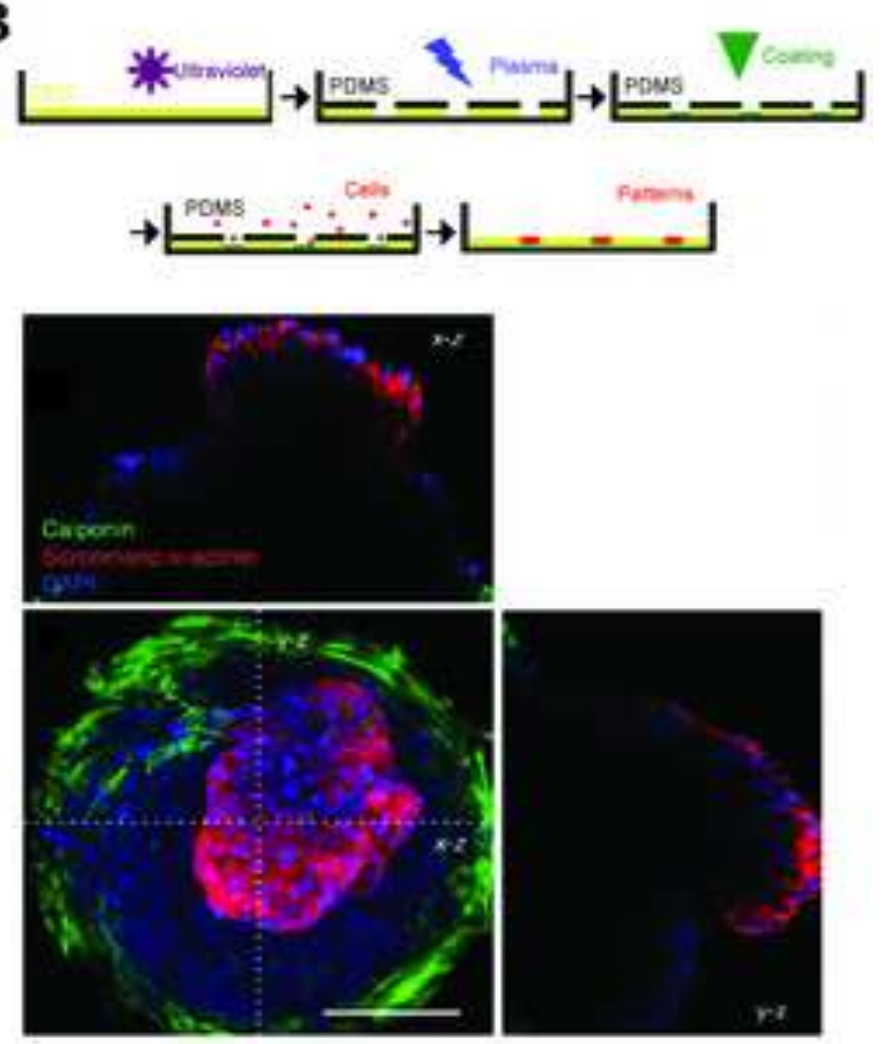